\title{
Stabilization of absolute instability in spanwise wavy two-dimensional wakes
}

\author{
Y ONG Y UN HWA NG ${ }^{1} \dagger$, JINSUNG KIM ${ }^{2}$, \\ AND HAECHEON CHOI
}

${ }^{1}$ Department of Applied Mathematics and Theoretical Physics, University of Cambridge, Centre for Mathematical Sciences, Wilberforce Road, Cambridge CB3 0WA, UK

${ }^{2}$ School of Mechanical and Aerospace Engineering, Seoul National University, Seoul 151-744, Korea

(Received 20 May 2013 and in revised form ??)

Controlling vortex shedding using spanwise varying passive or active actuation (namely three-dimensional control) has recently been reported as a very efficient method for regulating two-dimensional bluff-body wakes. However, the mechanism why and how the designed controller regulates vortex shedding has not been clearly understood. To understand this mechanism, we perform a linear stability analysis of two-dimensional wakes, of which the base flow is modified with the given spanwise waviness. Absolute and convective instabilities of the spanwise wavy base flows are investigated using Floquet theory. Two types of the base-flow modification are considered: varicose and sinuous modifications. Both of the base-flow modifications attenuate absolute instability of twodimensional wakes. In particular, the varicose modification is found much more effective in the attenuation than the sinuous one, and its spanwise lengths resulting in the maximum attenuation show good agreement with those in three-dimensional controls. The physical mechanism of the stabilization is found to be associated with formation of streamwise vortices from tilting of two-dimensional Kármán vortices and the subsequent tilting of these streamwise vortices by the spanwise shear in the base flow. Finally, the sensitivity of absolute instability to spanwise wavy base-flow modification is investigated. It is shown that absolute instability of two-dimensional wakes is much less sensitive to spanwise wavy base-flow modification than to two-dimensional modification. This suggests that the high efficiency observed in several three-dimensional controls is not due to sensitive response of wake instability to the spanwise waviness in base flow.

\section{Introduction}

The wake behind of a bluff body is an important canonical flow which we often encounter in many engineering applications. Vortex shedding in the near-wake region is an important source of drag, vibration, and noise generation, and therefore there have been numerous efforts for controlling it. Many of these efforts have often focused on developing control methods within two-dimensional framework: the control input is homogeneous in the spanwise direction (we shall refer to this approach as 'two-dimensional' control). The well-known examples include end plate (Nishioka \& Sato 1974; Stansby 1974), base bleed (Wood 1967; Bearman 1967), splitter plate (Roshko 1955; Bearman 1965; Kwon \& Choi 1996), secondary small cylinder (Strykowski \& Sreenivasan 1990), and active 
blowing/suction based with flow sensing (Min \& Choi 1999; Son et al. 2011). On the contrary, a number of relatively recent studies have proposed a conceptually different approach where the control input varies along the spanwise direction (we shall refer to this approach as 'three-dimensional' control). Segmented trailing edge (Tanner 1972; Rodriguez 1991), wavy trailing edge (Tombazis \& Bearman 1997), wavy front stagnation face (Bearman \& Owen 1998; Owen et al. 2000; Darekar \& Sherwin 2001), spanwise periodic blowing/suction (Kim et al. 2004; Kim \& Choi 2005), and small vertical tab (Park et al. 2006) are the examples which significantly attenuate vortex shedding in a wide range of the Reynolds number. For further details on this approach, the reader may refer to a recent review by Choi et al. (2008).

An important feature of the three-dimensional control is that it is often much more efficiently implemented than the two-dimensional one. For instance, base bleed, which injects a non-negligible amount of fluid at the rear of the cylinder, typically requires the actuation velocity $\phi \simeq 0.2 U_{\infty}$ ( $U_{\infty}$ is the free-stream velocity) for complete stabilization of vortex shedding (Schumm et al. 1994), while the spanwise wavy blowing and suction in Kim \& Choi (2005) achieve the same performance only with $\phi \simeq 0.07 U_{\infty}$. Despite of such high efficiency of this approach, the essential mechanism, how and why the threedimensional control regulates vortex shedding, remains poorly understood.

Hydrodynamic stability theories have experienced significant development for the last three decades, and they now provide a solid theoretical framework in understanding the onset and dynamics of vortex shedding in bluff-body wake. Vortex shedding is a typical nonlinear self-sustained oscillation which results from a supercritical Hopf bifurcation (Mathis et al. 1984; Provansal et al. 1987; Schumm et al. 1994). When the Reynolds number exceeds a critical value, a disturbance associated with unstable global modes undergoes exponential growth (Zebib 1987; Jackson 1987). The growing disturbance is then saturated due to stabilizing nonlinearity, and it eventually forms a limit-cycle oscillation. In weakly non-parallel flows, the appearance of such a global oscillation has been firmly associated with absolute instabilities in the near-wake region (Chomaz et al. 1988; Monkewitz 1988; Huerre \& Monkewitz 1990; Monkewitz et al. 1993; Pier 2002). More recent analyses have shown that this near-wake region acts as a wavemaker for both linear and nonlinear global instabilities (Chomaz 2005; Giannetti \& Luchini 2007; Hwang \& Choi 2008).

The hydrodynamic stability theory also provides important physical insights into the mechanism by which the given control strategy stabilizes vortex shedding. The control input often induces non-negligible amounts of change of base flow in the near-wake region acting as the wavemaker of vortex shedding. This change leads to stabilization of the near-wake absolute instability and subsequently of global instability. This mechanism has successfully explained why many two-dimensional controls yield stabilization of vortex shedding. For example, base bleed attenuates the near-wake absolute instability by weakening the strength of reverse flows in the recirculation zone (Monkewitz 1988; Hwang \& Choi 2006). Strong base suction, which promotes entrainment of the free-stream high momentum to the recirculation region, weakens the absolute instability in this region (Hammond \& Redekopp 1997; Leu \& Ho 2000). Placing a small secondary cylinder in the separating shear layer are also shown to stabilize both the near-wake absolute and global instabilities (Hwang \& Choi 2006; Marquet et al. 2008; Pralits et al. 2010).

The firm relation between the near-wake base-flow modification by the control input and the resulting stabilization of vortex shedding in many two-dimensional controls leads us to pose a key question for three-dimensional control: 'do the spanwise wavy base-flow modification by a three-dimensional control stabilize the near-wake absolute instability and global instability?'. We should emphasize that this question is actually tangled with 
two independent questions: 1) 'how does the designed control input modify the nearwake base flow?' and 2) 'does the spanwise waviness itself in the base flow play any role in stabilizing the near-wake absolute instability and/or global instability?'. Examining the two aspects of the given question is particularly important in understanding where the very high efficiency of several three-dimensional controls (Kim et al. 2004; Kim \& Choi 2005; Park et al. 2006) originates from: it could be from highly efficient base-flow modification by the given control input or from highly sensitive nature of wake instability itself to the spanwise waviness in base flow. The purpose of the present study is to provide appropriate answers to these questions arising from three-dimensional control. In particular, the present study is aimed at answering the latter by examining whether the presence of the spanwise waviness in base flow leads to stabilization of the near-wake absolute instabilities.

The presence of the spanwise waviness in base flow, however, yields a difficulty in performing the analysis that we aim. It has been viewed that theoretical investigation of spatio-temporal evolution of instabilities in spatially periodic base flows is a challenging issue. Although the criterion for absolute instability could be derived using Floquet theory (Brevdo \& Bridges 1996), its technical complexity has limited its application to only few cases for the linearized Navier-Stokes system (Bertolotti et al. 2004; Pier 2007). For this reason, most of previous studies examined the spatio-temporal evolution of instabilities using numerical simulation (Brancher \& Chomaz 1997; Brandt et al. 2003). However, the numerical simulation is often computationally very expensive because it requires very long streamwise domain which gives a space for the driven wave packets to evolve sufficiently far downstream. Therefore, when one is to examine relatively a large number of parameters as in the present study, this approach would not be suitable. This underlying difficulty forces us to follow the analytic approach proposed by Brevdo \& Bridges (1996). This therefore naturally poses another objective of the present study, which is to examine the analytic criterion of absolute instability in spatially periodic base flow for Navier-Stokes system.

The paper is organized as follows. In $\S 2$, we derive the analytic criterion of absolute instability for the streamwise parallel and spanwise periodic base flows. In $\S 3$, we numerically verify the criterion of absolute instability for two-dimensional parallel wakes containing a spanwise waviness in the base flow. We subsequently show that the base-flow modification with the given spanwise waviness results in stabilizing absolute instability. Discussion is then given in $\S 4$ where we present comparison of the present linear stability analysis with previous experimental and numerical studies for three-dimensional controls, physical mechanism of the stabilization, and sensitivity of absolute instability to the spanwise wavy-base flow modification compared with that to two-dimensional baseflow modification. A summary of the present work is given in $\S 5$ with several remarks.

\section{Problem formulation}

\subsection{Linear impulse response of streamwise parallel and spanwise periodic base flows}

We first analyze the impulse response of Navier-Stokes equation linearized around a streamwise parallel and spanwise periodic base flow. The streamwise parallel nature of the base flow allows us to use Fourier transform in this direction (e.g. Briggs 1964; Bers 1983; Huerre \& Monkewitz 1985, 1990; Huerre \& Rossi 1998), while in the spanwise direction, we use Floquet theory proposed by Brevdo \& Bridges (1996). It is convenient to start from the following form of the linearized Navier-Stokes equation (see Appendix 
A):

$$
\mathcal{M}\left(y ;-i \frac{\partial}{\partial x}\right) \frac{\partial \psi}{\partial t}+\frac{\partial \psi}{\partial z}=\mathcal{L}\left(y, z ;-i \frac{\partial}{\partial x}\right) \psi
$$

with the initial condition,

$$
\left.\psi\right|_{t=0}=\psi_{0}
$$

where $\psi(x, y, z, t)=\left[v \partial v / \partial z \partial^{2} v / \partial z^{2} \partial^{3} v / \partial z^{3} \eta \partial \eta / \partial z w \partial w / \partial z\right]^{T}, \eta$ is the transverse vorticity $(=\partial u / \partial z-\partial w / \partial x), x, y$, and $z$ respectively the streamwise, the transverse, and the spanwise directions, and $t$ the time. The linear operator $\mathcal{M}$ is constant in $z$, while $\mathcal{L}$ contains coefficients periodic in $z$ due the base flow considered: i.e.,

$$
\mathcal{L}\left(y, z ;-i \frac{\partial}{\partial x}\right)=\mathcal{L}\left(y, z+\lambda_{z} ;-i \frac{\partial}{\partial x}\right)
$$

where $\lambda_{z}$ is the spanwise period. Taking Laplace-Fourier transform of (2.1) in the time and the streamwise direction, $\hat{\psi}(y, z ; \alpha, \omega)=\int_{0}^{\infty} \int_{-\infty}^{\infty} \psi(x, y, z, t) e^{-i(\alpha x-\omega t)} d x d t$ ( $\alpha$ and $\omega$ are the streamwise wavenumber and the frequency, respectively), yields the following equation:

$$
\frac{\partial \hat{\psi}}{\partial z}=[\mathcal{L}(y, z ; \alpha)+i \omega \mathcal{M}(y ; \alpha)] \hat{\psi}+\mathcal{M}(y ; \alpha) \hat{\psi}_{0}
$$

It is convenient to assume that the transverse direction of (2.2) are projected onto a finite-dimensional vector space. In practice, the projection may be done with truncated series expansions or numerical discretization. This setting then enables us to use the techniques developed for finite-dimensional linear systems. We first introduce the state transition operator $\hat{\Psi}(z ; \alpha, \omega)$ of $(2.2)$ :

$$
\hat{\psi}(z ; \alpha, \omega)=\left.\hat{\Psi}(z ; \alpha, \omega) \hat{\psi}\right|_{z=0}
$$

where

$$
\frac{\partial \hat{\Psi}(z ; \alpha, \omega)}{\partial z}=[\mathcal{L}(z ; \alpha)+i \omega \mathcal{M}] \hat{\Psi}(z ; \alpha, \omega), \quad \hat{\Psi}(0 ; \alpha, \omega)=\mathcal{I}
$$

Here, $\mathcal{I}$ is the identity operator. We note that $\hat{\Psi}(z ; \alpha, \omega)$ is non-singular (see e.g. Antsaklis \& Michel 1997).

Now, we observe that $\mathcal{L}$ is continuous and periodic matrix function in $z$. Therefore, Floquet's theorem gives the following form of the state transition operator $\hat{\Psi}(z ; \alpha, \omega)$ :

$$
\hat{\Psi}(z ; \alpha, \omega)=\hat{\mathcal{Q}}(z ; \alpha, \omega) e^{z \mathcal{B}(\alpha, \omega)},
$$

where

$$
\hat{\mathcal{Q}}(z ; \alpha, \omega)=\hat{\mathcal{Q}}\left(z+\lambda_{z} ; \alpha, \omega\right), \quad \hat{\mathcal{Q}}(0 ; \alpha, \omega)=\mathcal{I} .
$$

Here, the operator $\mathcal{B}$ in the matrix exponential of $(2.4 a)$ describes the spanwise evolution of the solution of (2.1), and its eigenvalues are referred to as Floquet exponents. Since $\hat{\Psi}(z ; \alpha, \omega)$ is not singular, it is also evident that $\hat{\mathcal{Q}}$ is non-singular and invertible.

Equation $(2.4 a)$ suggests that $\hat{\psi}$ is in a subspace of range of $\hat{\mathcal{Q}}$, which allows us to write $\hat{\psi}$ as follows:

$$
\hat{\psi}(z ; \alpha, \omega)=\hat{\mathcal{Q}}(z ; \alpha, \omega) \hat{\phi}(z ; \alpha, \omega) .
$$

Then, combining $(2.2),(2.3 b),(2.4 a)$, and (2.5) all together gives

$$
\frac{\partial \hat{\phi}(z ; \alpha, \omega)}{\partial z}-\mathcal{B}(\alpha, \omega) \hat{\phi}(z ; \alpha, \omega)=\hat{\mathcal{Q}}^{-1} \mathcal{M} \hat{\psi}_{0}
$$

We now note that (2.6) turns out to be a $z$-invariant linear system. Therefore, this enables 
us to use the Fourier transform in $z: \tilde{\phi}(\alpha, \beta, \omega)=\int_{-\infty}^{\infty} \hat{\phi}(z ; \alpha, \omega) e^{-i \beta z} d z$, where $\beta$ is the spanwise wavenumber. This yields

$$
\tilde{\phi}(\alpha, \beta, \omega)=(i \beta \mathcal{I}-\mathcal{B})^{-1} \tilde{h}_{0},
$$

where

$$
\tilde{h}_{0} \equiv \int_{-\infty}^{\infty} \hat{\mathcal{Q}}^{-1} \mathcal{M} \tilde{\psi}_{0} e^{-i \beta z} d z
$$

The physical-space solution $\psi(x, z, t)$ is then built by inversion of the Laplace-Fourier transform:

$$
\psi(x, z, t)=\frac{1}{(2 \pi)^{3}} \int_{B_{\omega}} \int_{C_{\alpha}} \hat{\mathcal{Q}} \int_{C_{\beta}} \frac{\mathcal{S}(\alpha, \beta, \omega)}{\mathcal{D}(\alpha, \beta, \omega)} e^{i(\alpha x+\beta z-\omega t)} d \beta d \alpha d \omega,
$$

where

$$
\begin{gathered}
\mathcal{S}(\alpha, \beta, \omega)=\operatorname{det}[i \beta \mathcal{I}-\mathcal{B}](i \beta \mathcal{I}-\mathcal{B})^{-1} \tilde{h}_{0}, \\
\mathcal{D}(\alpha, \beta, \omega)=\operatorname{det}[i \beta \mathcal{I}-\mathcal{B}(\alpha, \omega)] .
\end{gathered}
$$

Here, $B_{\omega}, C_{\alpha}$, and $C_{\beta}$ are respectively integral contours in the complex $\omega_{-}, \alpha_{-}$, and $\beta$ - planes for the inverse Laplace-Fourier transform, and they are shown in Figs. $1(a)$, $(d)$, and $(g)$ respectively. We note that the integrand in $(2.8 a)$ becomes singular when $\mathcal{D}(\alpha, \beta, \omega)=0$, implying that this gives dispersion relation of (2.1).

The asymptotic solution of the integral (2.8a) is obtained using the method of steepest descent (Briggs 1964; Huerre \& Monkewitz 1985, 1990; Huerre \& Rossi 1998) that was also adopted in Carriere \& Monkewitz (1999) for the evaluation of an integral similar to $(2.8 a)$. Following the same procedure in Carriere \& Monkewitz (1999), the asymptotic solution of $(2.8 a)$ for sufficiently large $t$ is obtained as

$$
\begin{aligned}
& \psi(x, z, t) \sim \\
& t^{-1}\left[\frac{\partial^{2} \omega}{\partial \alpha^{2}}\left(\alpha_{0}, \beta_{0}\right) \frac{\partial^{2} \omega}{\partial \beta^{2}}\left(\alpha_{0}, \beta_{0}\right)\right]^{-1 / 2} \hat{\mathcal{Q}}\left(z ; \alpha_{0}, \omega_{0}\right) \frac{\mathcal{S}\left(\alpha_{0}, \beta_{0}, \omega_{0}\right)}{\frac{\partial \mathcal{D}}{\partial \omega}\left(\alpha_{0}, \beta_{0}, \omega_{0}\right)} e^{i\left(\alpha_{0} x+\beta_{0} z-\omega_{0} t\right)},
\end{aligned}
$$

where

$$
\frac{\partial \omega}{\partial \alpha}\left(\alpha_{0}, \beta_{0}\right)=\frac{\partial \omega}{\partial \beta}\left(\alpha_{0}, \beta_{0}\right)=0 \quad \text { and } \quad \omega_{0}=\omega\left(\alpha_{0}, \beta_{0}\right) .
$$

Here, $\alpha_{0}$ and $\beta_{0}$ are respectively complex streamwise and spanwise absolute wavenumbers, and $\omega_{0}$ is complex absolute frequency. The absolute wavenumbers, $\alpha_{0}$ and $\beta_{0}$, characterize the dominant spatial wavelength of the initially driven wave packet as $t \rightarrow \infty$, and the absolute frequency $\omega_{0}$ gives frequency and growth rate of the wave packet along the rays of $x / t=0$ and $z / t=0$. We note that, in (2.10), $\partial \omega / \partial \beta\left(\alpha_{0}, \beta_{0}\right)=0$ additionally appears compared to the two-dimensional parallel flow case ( e.g. Huerre \& Monkewitz 1985, 1990; Huerre \& Rossi 1998; Chomaz 2005). This is essentially due to the threedimensionality considered here: in three-dimensional flows, the localized instability wave packet should exhibit growth along the ray of $z / t=0$ as well as the ray of $x / t=0$ to be absolutely unstable.

In practice, it is convenient to use a criterion equivalent to (2.10) in terms of the so-called monodromy operator $\Psi\left(\lambda_{z} ; \alpha, \omega\right)\left(=e^{\lambda_{z} \mathcal{B}}\right)$, which describes the evolution of $\psi$ over a spanwise period $\lambda_{z}$ (see also section 2.3). Introducing the spanwise wavenumber associated with the monodromy operator, $\mu \equiv e^{i \beta \lambda_{z}}$, gives the following criterion of absolute instability:

$$
\frac{\partial \omega}{\partial \alpha}\left(\alpha_{0}, \mu_{0}\right)=\frac{\partial \omega}{\partial \mu}\left(\alpha_{0}, \mu_{0}\right)=0 \quad \text { and } \quad \omega_{0}=\omega\left(\alpha_{0}, \mu_{0}\right) .
$$


(a)

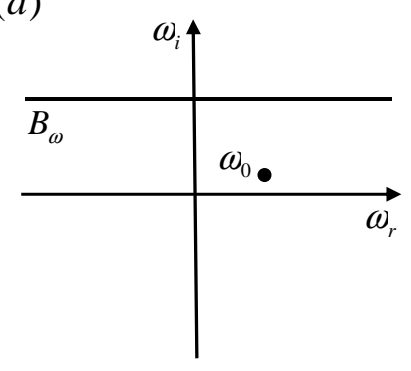

(b)

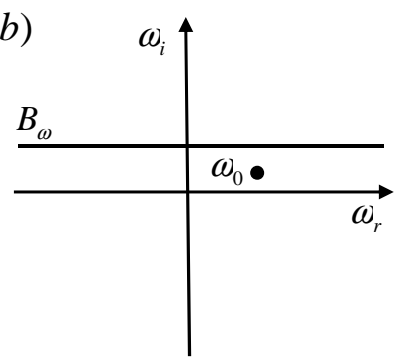

(c)

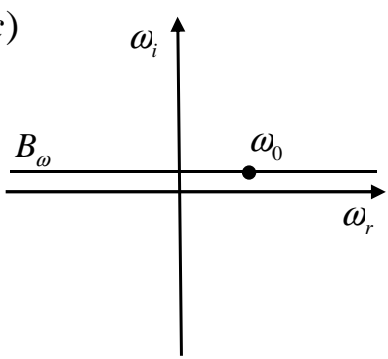

$(d)$

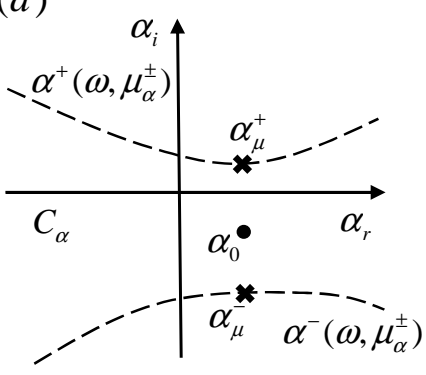

(e)
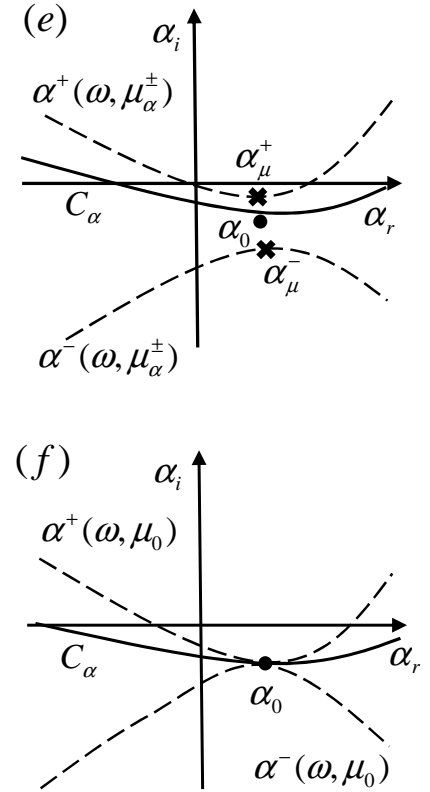

$(g)$

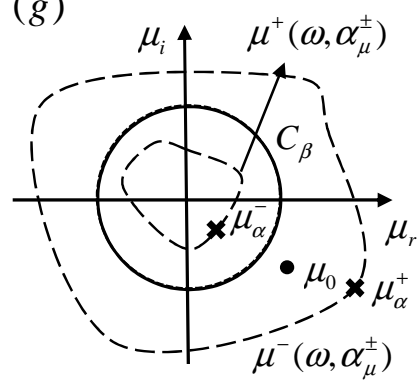

(h)

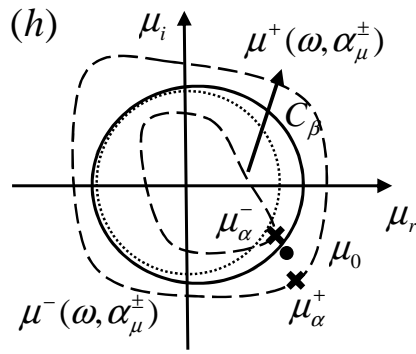

(i)

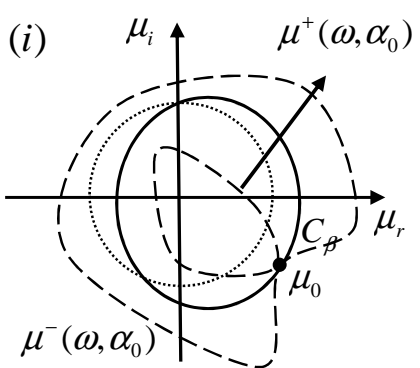

Figure 1. Lowering of the Bromwich contour $B_{\omega}$ in the complex $\omega$-plane, and the corresponding locus of spatial branches in the complex $\alpha$ - and $\mu$-planes (from top to bottom). Here, $(a-c) \omega-$, $(d-f) \alpha-$, and $(g-i) \mu$-planes. In $(g-i)$, the dotted circle indicates $|\mu|=1$.

It should be emphasized that the criterion in (2.10) or (2.11), which satisfies zero complex-group-velocity condition, is the so-called 'pinching' point (Briggs 1964; Huerre \& Monkewitz 1985, 1990; Huerre \& Rossi 1998). The pinching point could be rigorously detected by gradually lowering the Bromwhich contour $B_{\omega}$, as shown in figure 1 . We note that the pinching point detection procedure described here is an extension of the standard procedure for two-dimensional parallel base flows to that for spanwise periodic and streamwise parallel ones. For the details on the two-dimensional parallel cases, the reader may refer to several reviews (e.g. Huerre \& Monkewitz 1990; Huerre \& Rossi 1998). For illustrative purpose, here we assume that, for a given $\omega$, the dispersion relation gives two streamwise $\left(\alpha^{+}\right.$and $\left.\alpha^{-}\right)$and two spanwise $\left(\mu^{+}\right.$and $\left.\mu^{-}\right)$spatial branches. First, we imagine that $B_{\omega}$ is located sufficiently far above the pinching point $\omega_{0}$ in the $\omega$-plane (figure 1a). For a given $\mu\left(=\mu_{\alpha}^{+}\right.$or $\left.\mu_{\alpha}^{-}\right)$, the images of $B_{\omega}$ in the $\alpha$-plane form two streamwise spatial branches that are well separated from each other (the dashed lines in figure $1 d$ ). This allows the contour $C_{\alpha}$ to safely pass along the real axis in the $\alpha$ plane without violating causality for proper evaluation of (2.8a) (the solid line in figure 
$1 d$ along the real axis). Similarly, the images of $B_{\omega}$ for a given $\alpha\left(=\alpha_{\mu}^{+}\right.$or $\left.\alpha_{\mu}^{-}\right)$in the complex $\mu$-plane appear as two well-separated spanwise spatial branches. One of them for $z<0\left(\mu^{-}\right)$is located inside of the unit circle $(|\mu|=1)$ and the other for $z>0$ $\left(\mu^{+}\right)$is placed outside of the circle by the definition of $\mu\left(\equiv e^{i \beta \lambda_{z}}\right)$ (figure $\left.1 g\right)$. The image of integration contour $C_{\beta}$, which is a unit circle in the $\mu$-plane (solid line in figure $1 g$ ), therefore also safely passes through the space between two branges without violating causality. Now, we gradually lower the $B_{\omega}$ toward the pinching point $\omega_{0}$ (figure $1 b$ ). As $B_{\omega}$ is lowered, the two spatial branches in the $\alpha$-plane $\left(\alpha^{+}\right.$and $\left.\alpha^{-}\right)$become closer to each other. In this circumstance, to enforce the causality condition for evaluating $(2.8 a)$, the integral contour $C_{\alpha}$ in the $\alpha$-plane should be deformed so that it passes through the space between $\alpha^{+}$and $\alpha^{-}$branches (figure 1e). Similarly, in the complex $\mu$-plane, the image of the integration contour $C_{\beta}$ should also be deformed so that it lies on the space between $\mu^{+}$and $\mu^{-}$branches, which approach each other with lowering $B_{\omega}$ (figure $1 h$ ). When $B_{\omega}$ eventually passes through the pinching point $\omega_{0}$ due to its lowering (figure $1 c$ ), the two branches in the $\alpha$-plane meets each other at $\alpha=\alpha_{0}$ (figure $1 f$ ) and those in the $\mu$-planes also shows the same behavior at $\mu=\mu_{0}$ (figure $1 i$ ). It should be emphasized that $B_{\omega}$ is not able to be further lowered for evaluation of $(2.8 a)$ because this leads to breakdown of the causality. Finally, we note that the local topology of $\omega_{i}$ near the pinching point in both the complex $\alpha$ - or $\mu$-planes forms saddle, which also allows one to use the method of steepest descent for asymptotic evaluation of (2.8a) as in (2.9).

\subsection{Equations of motion}

As shown in appendix A, the linearized equations of motion for the base flow, $(U(y, z)=$ $\left.U\left(y, z+\lambda_{z}\right), 0,0\right)$, are the following:

$$
\begin{gathered}
\frac{\partial u}{\partial x}+\frac{\partial v}{\partial y}+\frac{\partial w}{\partial z}=0 \\
\frac{\partial u}{\partial t}+U \frac{\partial u}{\partial x}+v \frac{\partial U}{\partial y}+w \frac{\partial U}{\partial z}=-\frac{\partial p}{\partial x}+\frac{1}{R e} \nabla^{2} u \\
\frac{\partial v}{\partial t}+U \frac{\partial v}{\partial x}=-\frac{\partial p}{\partial y}+\frac{1}{R e} \nabla^{2} v \\
\frac{\partial w}{\partial t}+U \frac{\partial w}{\partial x}=-\frac{\partial p}{\partial z}+\frac{1}{R e} \nabla^{2} w
\end{gathered}
$$

where $u, v$, and $w$ are respectively the streamwise, transverse, and spanwise velocity perturbations, and $p$ is the pressure perturbation. Since the scope of the present study is to understand the role of the given spanwise waviness in absolute instability of twodimensional wakes, we consider a simplest description of the base flow by assuming that it is composed of a two-dimensional wake profile and a base-flow modification with a single spanwise wave component:

$$
U(x, y, z)=U_{0}(y)+A \cdot U_{1}(y) \cos \left(2 \pi \frac{z}{\lambda_{z}}\right),
$$

where $U_{0}(y)$ is the profile of typical two-dimensional wake, $A$ the amplitude of the baseflow modification, and $U_{1}(y)$ the normalized shape function for the modification. We note that the description by (2.13) may be a 'minimal' representation of the base flow modified by three-dimensional control (see also discussion in section 4.1). From the base-flow profile in (2.13), velocities in (2.12) are non-dimensionalized by $\bar{U}_{0}^{*}\left(=\left(U_{c}^{*}+U_{\infty}^{*}\right) / 2\right)$ (the superscript ${ }^{*}$ denotes a dimensional quantity), where $U_{c}^{*}$ and $U_{\infty}^{*}$ are the centerline and free-stream velocities, respectively. The lengths are made non-dimensional with the wake 


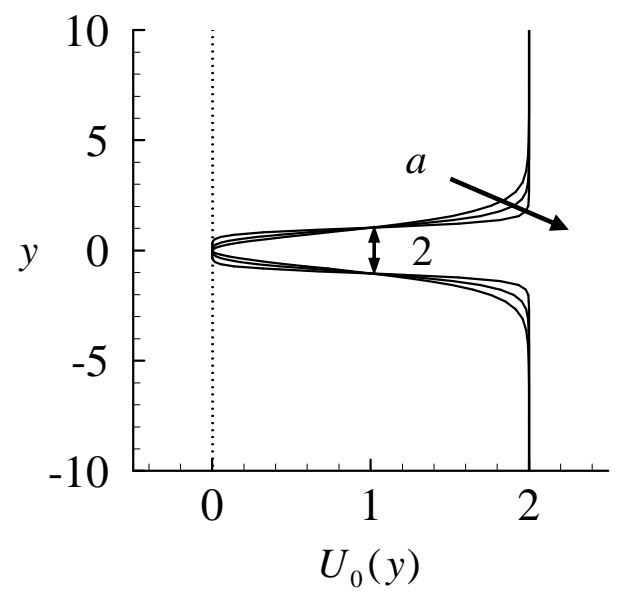

Figure 2. Profiles of the model parallel wake $U_{0}(y)$ for $a=1,1.5,3(\Lambda=-1)$.

half-width $h$ such that $\left.U^{*}\right|_{y^{*}=h}=\bar{U}_{0}^{*}$. The non-dimensionalization gives the Reynolds number as $R e=\bar{U}_{0}^{*} h / \nu$.

For $U_{0}(y)$ in (2.13), we consider a symmetric model wake proposed by Monkewitz (1988). The wake profile is given as

$$
U_{0}(y)=1-\Lambda+2 \Lambda F(y),
$$

where

$$
\begin{gathered}
\Lambda=\left(U_{c}^{*}-U_{\infty}^{*}\right) /\left(U_{c}^{*}+U_{\infty}^{*}\right), \\
F(y)=\left[1+\sinh ^{2 a}\left\{y \sinh ^{-1}(1)\right\}\right]^{-1} .
\end{gathered}
$$

Here, $\Lambda$ is a parameter controlling the depth of the wake and setting $\Lambda=-1$ generates the profiles with zero centerline velocity. The parameter $a \in[1, \infty)$ determines the ratio of shear-layer thickness to wake width, and $a=\infty$ and $a=1$ correspond to a top-hat wake and the standard $\operatorname{sech}^{2} y$ wake, respectively. The profiles of $U_{0}(y)$ for a few $a$ 's are shown in figure. 2 .

For the normalized shape function for the modification $U_{1}(y)$, we consider the following two profiles:

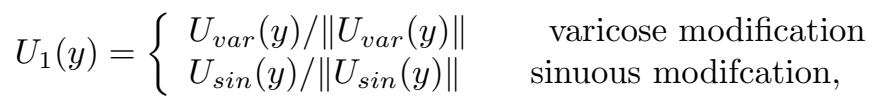

where

$$
\begin{aligned}
& U_{v a r}(y)=e^{\frac{-(y-\xi)^{2}}{2 \sigma^{2}}}+e^{\frac{-(y+\xi)^{2}}{2 \sigma^{2}}} \\
& U_{s i n}(y)=e^{\frac{-(y-\xi)^{2}}{2 \sigma^{2}}}-e^{\frac{-(y+\xi)^{2}}{2 \sigma^{2}}}
\end{aligned}
$$

Here, $\|\cdot\| \equiv \sqrt{\int_{-\infty}^{\infty}(\cdot)^{2} d y}$, and $\sigma$ and $\xi$ are parameters determining the width of the Gaussian and the distance between upper and lower extrema, respectively. We note that these profiles are chosen from the control configurations in our previous studies (Kim et al. 2004; Kim \& Choi 2005; Park et al. 2006). For example, Kim et al. (2004) and Kim \& Choi (2005) considered a spanwise sinusoidal blowing and suction applied at upper and lower surfaces of a given bluff body. In particular, they introduced two control strategies, one of which has no phase difference between the blowing and suction profiles at the two slots (in-phase forcing) and the other has $\pi$ (out-of-phase forcing). 

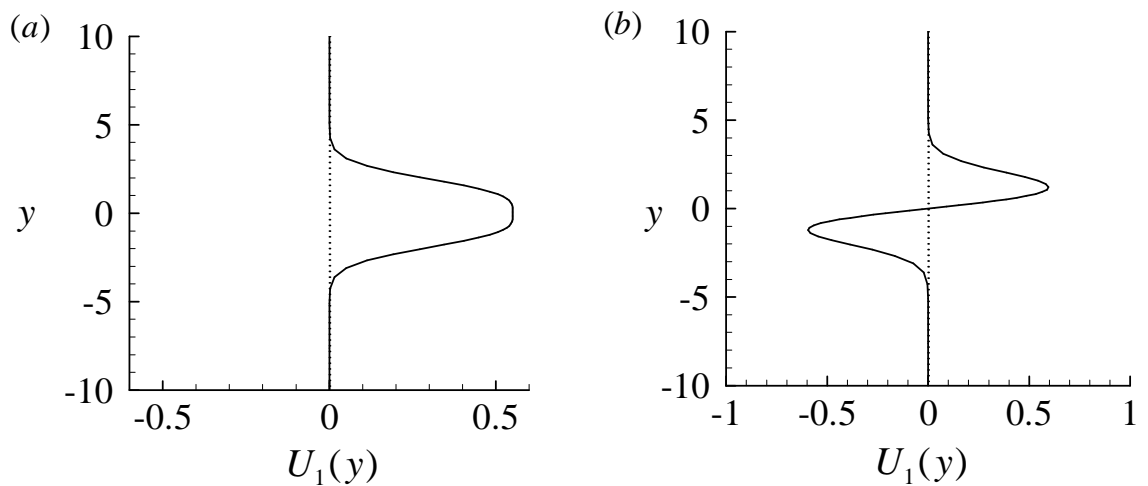

Figure 3. Profiles of the normalized shape functions for base-flow modification $U_{1}(y)(\sigma=1$ and $\xi=1)$ : $(a)$ varicose and $(b)$ sinuous modifications.

A similar configuration was also considered in Park et al. (2006) in which the control is implemented by small vertical tabs instead of the blowing and suction. In the present study, we design the varicose modification to mimic the change of the near-wake base flow by the in-phase forcing in Kim \& Choi (2005), while the sinuous modification is designed to describe the change by the out-of-phase forcing. The profiles of $U_{1}(y)$ considered for varicose and sinuous modifications are respectively presented in figures $3(a)$ and $(b)$.

We finally note that the base-flow profile described by (2.13) contains many control parameters. Therefore, to minimize the number of parameters, some of them are set to be fixed throughout the entire paper. First, the parameter controlling the depth of the unmodified base flow $U_{0}(y)$ is fixed as $\Lambda=-1$ so that the centerline velocity of $U_{0}(y)$ becomes zero. We also fix $\sigma=1$ and $\xi=1$ for $U_{1}(y)$ from observations of the near-wake base flows in one of the previous numerical experiments (Kim \& Choi 2005; Kim 2005).

\subsection{Numerical methods}

The dispersion relation of (2.12) is computed by formulating the transverse-velocity $(v)$ and vorticity $(\eta)$ form (see also Appendix A). In this case, the normal-mode solution is given as follows:

$$
\begin{aligned}
& v(x, y, z, t)=e^{i(\alpha x+\beta z-\omega t)} \underbrace{\sum_{n=-N_{z} / 2}^{N_{z} / 2} \widetilde{v}_{n}(y) e^{i n \frac{2 \pi}{\lambda z} z}}_{\hat{q}_{v}(y, z)}+c . c, \\
& \eta(x, y, z, t)=e^{i(\alpha x+\beta z-\omega t)} \underbrace{\sum_{n=-N_{z} / 2}^{N_{z} / 2} \tilde{\eta}_{n}(y) e^{i n \frac{2 \pi}{\lambda z} z}}_{\hat{q}_{\eta}(y, z)}+c . c,
\end{aligned}
$$

where $\widetilde{v}_{n}(y)$ and $\widetilde{\eta}_{n}(y)$ are respectively the Fourier modes of the spanwise periodic variables $\hat{q}_{v}(y, z)$ and $\hat{q}_{\eta}(y, z)$, and $N_{z}$ is the number of the expansion coefficients. For $N_{z} \rightarrow \infty$, the periodic nature of $\hat{q}_{v}$ and $\hat{q}_{\eta}$ in the spanwise direction implies that the dispersion relation for $\beta \in\left[-\pi / \lambda_{z}, \pi / \lambda_{z}\right)$ is identical to that for $\beta \in\left[n \pi / \lambda_{z},(n+2) \pi / \lambda_{z}\right)$ ( $n$ is an integer). Introducing the spanwise wavenumber associated with the monodromy operator $\left(\mu=e^{i \beta \lambda_{z}}\right)$ enables us to map the dispersion relation in the complex $\beta$-plane, 

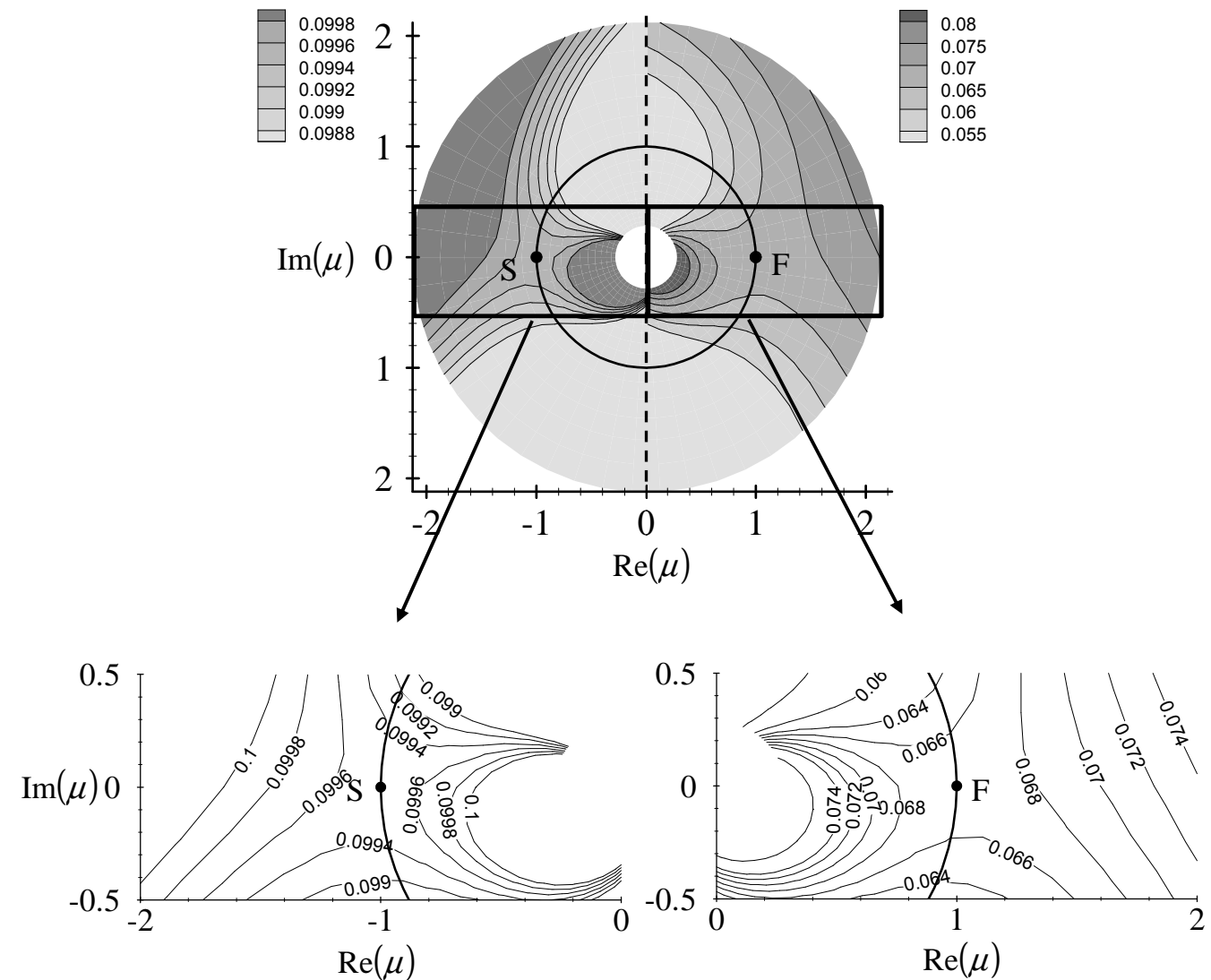

Figure 4. Topology of $\omega_{i}\left(\alpha_{0}, \mu\right)$ in the complex $\mu$ plane with varicose base-flow modification $(a=1.5, A=0.3$, and $R e=50)$. The left and right halves of the contour are respectively shown for $\lambda_{z}=10\left(\alpha_{0}=0.893-i 0.559\right)$ and $\lambda_{z}=6\left(\alpha_{0}=0.813-i 0.584\right)$. The circle with thick solid line indicates $|\mu|=1$. Two saddle points appear at $\mu=1$ (fundamental mode) and $\mu=-1$ (subharmonic mode).

which repeats over with the period $2 \pi / \lambda_{z}$, onto the entire complex $\mu$-plane, resulting in a dispersion relation uniquely defined in the entire complex $\mu$-plane.

The normal-mode solution is computed by formulating a temporal eigenvalue problem: $\omega$ is the eigenvalue for given complex $\alpha$ and $\mu$. The transverse direction is discretized using the standard Chebyshev-collocation method (Canuto et al. 1988). The resulting discretized eigenvalue problems are then solved using Matlab. Most of the computation in the present study is carried out with $N_{y} \times\left(N_{z}+1\right)=66 \times 9$. The results for several cases have been checked with $N_{y} \times\left(N_{z}+1\right)=100 \times 13$, showing no difference from those with the lower resolution. We note that the relatively small number of the grid points in the spanwise direction is due to the fact that the base flow given by (2.13) contains only a single spanwise wave component. For the details, see also Appendix $\mathrm{C}$ in which we examine the effect of the spanwise grid point $N_{z}$. 


\section{Results}

\subsection{The saddle points in complex wavenumber planes}

To seek applicability of the criterion (2.11) with the computed dispersion relation, we first study topology of growth rate $\omega_{i}$ in the complex $\mu$-plane. Figure 4 shows $\omega_{i}(\alpha, \mu)$ in the complex $\mu$ plane for two sets of given parameters (the left and right halves, respectively). For a given set of the parameters, two saddle points are found in the complex $\mu$-plane, and they are located at $\mu= \pm 1$ respectively. Several different sets of the parameters are also tested, but the choice of the parameters does not change the location of two saddle points in the complex $\mu$-plane. Furthermore, at least for the parameters tested, the saddle points in the complex $\mu$-plane appear only at these locations. The appearance of the two saddle points at $\mu= \pm 1$ is an interesting feature because they are respectively the so-called fundamental $(\mu=1)$ and subharmonic $(\mu=-1)$ modes in the temporal stability analysis using Floquet theory (Herbert 1988; Reddy et al. 1998). However, given the fact that the governing system does not have any biased spanwise advection by base flow, this may not be so surprising.

The location of the two saddle points in the complex $\mu$-plane can be analytically derived using the properties of dispersion relation of the governing system. For convenience, here we use the spanwise wavenumber $\beta$ instead of $\mu$ for the dispersion relation. As we discussed in section 2.3, dispersion relation for $\beta \in\left[-\pi / \lambda_{z}, \pi / \lambda_{z}\right)$ is identical to that for $\beta \in\left[n \pi / \lambda_{z},(n+2) \pi / \lambda_{z}\right)$ : i.e.

$$
\omega(\alpha, \beta)=\omega\left(\alpha, \beta+2 \pi / \lambda_{z}\right)
$$

The governing system (2.12) also exhibits even symmetry in the spanwise direction: $u(x, y, z, t)=u(x, y,-z, t), v(x, y, z, t)=v(x, y,-z, t), w(x, y, z, t)=-w(x, y,-z, t)$, and $p(x, y, z, t)=p(x, y,-z, t)$ (see also Schoppa \& Hussain 2002). This leads to the following property of the dispersion relation:

$$
\omega(\alpha, \beta)=\omega(\alpha,-\beta) .
$$

Using (3.1a) and (3.1b), it is straightforward to show $\partial \omega /\left.\partial \beta\right|_{\beta=0}=\partial \omega /\left.\partial \beta\right|_{\beta=\pi / \lambda_{z}}=0$. The equivalent relations in the complex $\mu$-plane are given as

$$
\left.\frac{\partial \omega}{\partial \mu}\right|_{\mu= \pm 1}=0 \text {. }
$$

This explains why the saddle points in the complex $\mu$-plane appear at $\mu= \pm 1$ independently of the choice of the parameters.

The appearance of saddle points at $\mu_{0}= \pm 1$ in the complex $\mu$-plane greatly simplifies the searching process of the saddle point (2.11) defined in both the complex $\alpha$ - and $\mu$-planes. Since the spanwise absolute wavenumber is always given by either $\mu_{0}=1$ or $\mu_{0}=-1$, one just needs to search the saddle point only in the complex $\alpha$-plane by fixing $\mu_{0}$ at either one of them. Several methods for detecting the saddle point in a complex plane are available, and, in the present study, we use the secant method with the initial guess guided by the cusp-map procedure (Schmid \& Henningson 2001). Figure 5 shows the topology of $\omega_{i}\left(\alpha, \mu_{0}\right)$ around a saddle point in the complex $\alpha$-plane with the given $\mu_{0}$. For both $\mu_{0}= \pm 1$, a saddle point in the complex $\alpha$-plane is clearly found. Once the two absolute frequencies $\omega_{0}$ are found with $\mu_{0}= \pm 1$, the most unstable absolute mode is then determined by comparing their growth rates with each other. In a certain range of the parameters, the two absolute modes are found to strongly compete with each other, but the detailed discussion on this issue is given in section 3.2. 

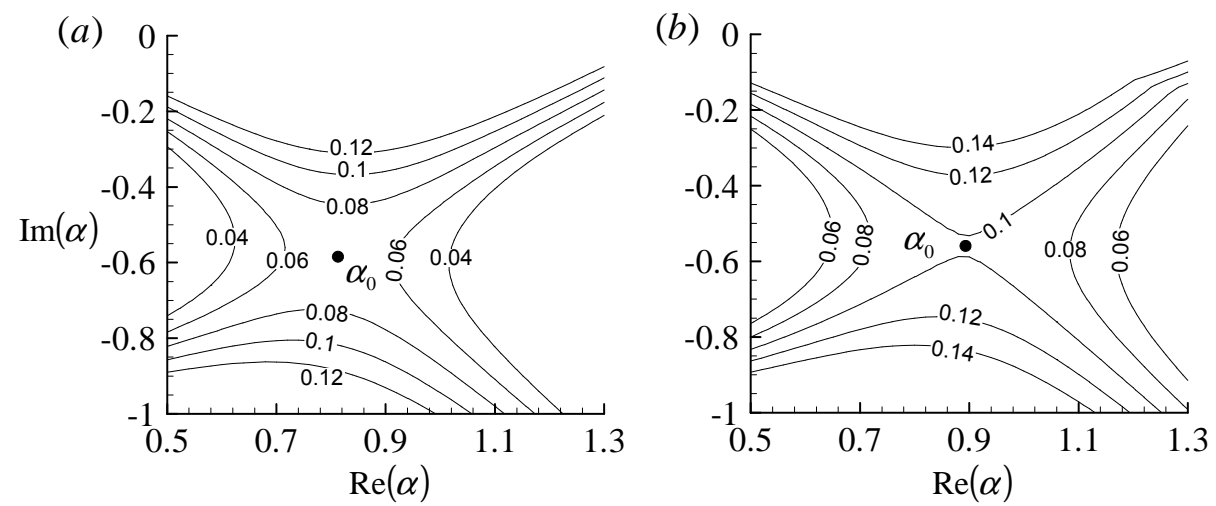

Figure 5. Topology of $\omega_{i}\left(\alpha, \mu_{0}\right)$ in the complex $\alpha$ plane with varicose base-flow modification $(a=1.5, A=0.3$, and $R e=50):(a) \lambda_{z}=6\left(\mu_{0}=1, \alpha_{0}=0.813-i 0.584\right) ;(b) \lambda_{z}=10$ $\left(\mu_{0}=-1, \alpha_{0}=0.893-i 0.559\right)$.
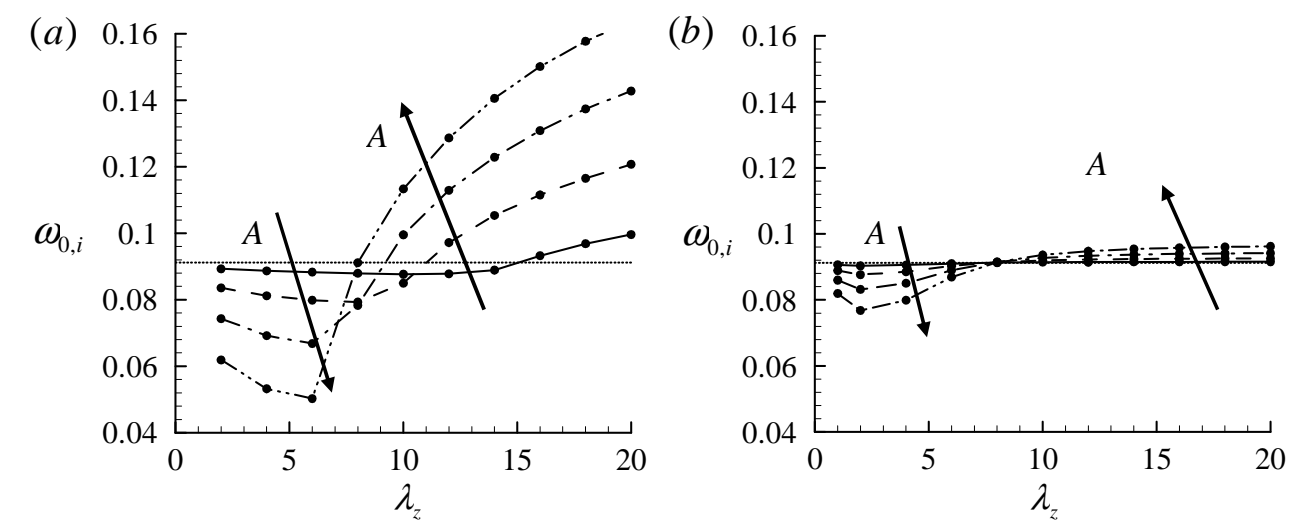

FIGURE 6 . Absolute growth rate $\omega_{0, i}$ with respect to the spanwise wavelength and the amplitude of the base-flow modifications $(a=1.5$ and $R e=50):(a)$ varicose and $(b)$ sinuous modifications. Here, $\cdots \cdots \cdots, A=0 ;-, A=0.1 ;-\cdots,-\cdot A=0.2 ;-\cdot-\cdot-, A=0.3 ;-\cdots-\cdots, A=0.4$.

\subsection{The spanwise wavelength and amplitude of base-flow modifications}

Using the procedure of finding the saddle points (2.11) given in the previous section, the response of absolute instability to the spanwise waviness in base flow is tested for a number of different sets of the parameters. Figure $6(a)$ reports the change of $\omega_{0, i}$ with the spanwise wavelength and the amplitude of varicose base-flow modification. For small amplitudes, varicose base-flow modification attenuates absolute instability in wide ranges of relatively small $\lambda_{z}(<10-15)$, while the instability exhibits destabilization for large $\lambda_{z}$. As the amplitude $A$ increases, the stabilizing effect becomes more pronounced particularly at small $\lambda_{z}(<6-8)$. However, the range of $\lambda_{z}$ attenuating absolute instability becomes narrower and the $\lambda_{z}$ providing the maximum stabilization also gradually becomes smaller. For large $\lambda_{z}$, the varicose modification significantly destabilizes absolute instability with the increase of the amplitude $A$. Similar tendency is also found for sinuous base-flow modification as shown in figure $6(b)$. However, in this case, the amount of change in $A$ is much smaller than that in the case of varicose modification. Also, the $\lambda_{z}$ yielding the maximum stabilization is found much shorter than that in varicose modification, and it changes very little with the increase of $A$. 

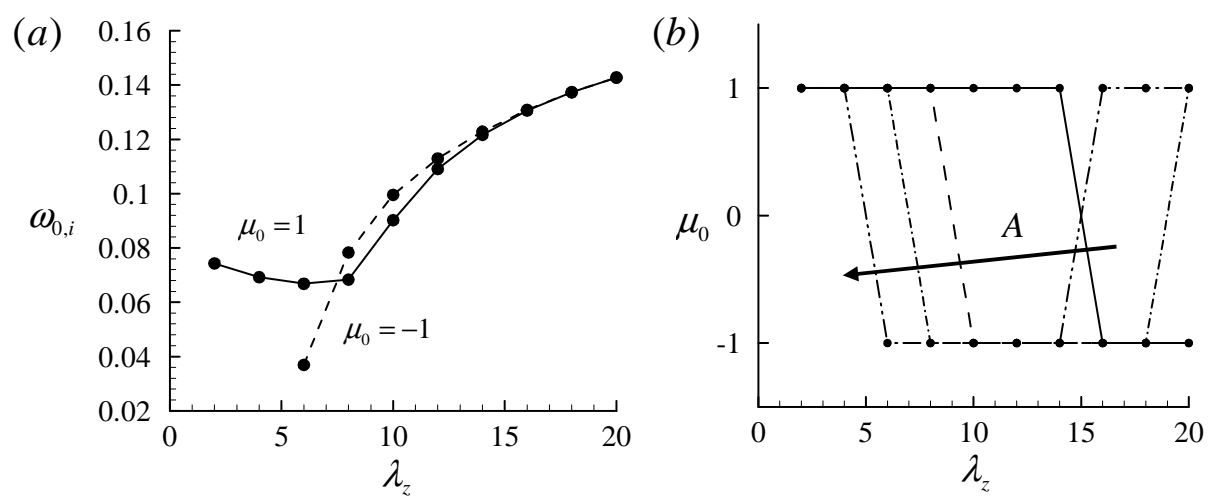

Figure 7. Competition between fundamental $\left(\mu_{0}=1\right)$ and subharmonic $\left(\mu_{0}=-1\right)$ modes in varicose base-flow modification $(a=1.5$ and $R e=50)$ : $(a)$ absolute growth rate $\omega_{0, i}$ of the fundamental (- $)$ and subharmonic $(---)$ modes with respect to the spanwise wavelength for $A=0.3 ;(b)$ absolute spanwise wavenumber $\mu_{0}$ of the most unstable mode with respect to the spanwise wavelength and the amplitude. In $(b),-\longrightarrow, A=0.1 ;----, A=0.2 ;-\cdot--$, $A=0.3 ;-\cdots-\cdots-, A=0.4$.

For varicose base-flow modification, the two absolute modes respectively from $\mu_{0}= \pm 1$ are found to highly compete with each other. Figure $7(a)$ shows comparison between absolute growth rates of the fundamental $\left(\mu_{0}=1\right)$ and subharmonic $\left(\mu_{0}=-1\right)$ modes. For the short spanwise wavelengths $\left(\lambda_{z}<7\right)$, the fundamental mode exhibits much larger growth rate than the subharmonic one. The subharmonic mode experiences significant destabilization with the increase of $\lambda_{z}$, and it becomes more unstable than the fundamental mode at $\lambda_{z} \simeq 7$. However, the difference between the growth rates of the two modes quickly diminishes as the spanwise wavelength increases. For sufficiently large $\lambda_{z}(\gtrsim 10)$, the fundamental mode again becomes slightly more unstable than the subharmonic one. As shown in figure $7(b)$, this tendency is also found for all the amplitudes considered. In general, with the increase of the amplitude $A$, the spanwise wavelength $\lambda_{z}$, at which the switch of the most unstable mode appears (either from the fundamental to subharmonic or from the subharmonic to fundamental), becomes gradually shorter (figure $7 b$ ). Finally, it should be emphasized that this competition between the fundamental and subharmonic modes appears only for varicose base-flow modification. In the case of sinuous modification, the most unstable mode is found as the fundamental mode $\left(\mu_{0}=1\right)$ for all the parameters tested in the present study.

\subsection{Eigenfunctions}

The spatial structures of eigenfunctions of absolute modes are visualized. In particular, we focus on showing the transverse and spanwise velocity perturbation, that are found to play an important role in dynamics of vorticity perturbations (see section 4.2). Figure 8 shows the transverse and spanwise velocity perturbations of the fundamental-mode absolute instability $\left(\mu_{0}=1\right)$ for varicose base-flow modification. For all the $\lambda_{z}$ considered, the transverse velocity perturbation of the absolute mode is relatively intense near $y=0$ (figures $8 a, c, e)$. For the smallest $\lambda_{z}(=2)$, the transverse velocity perturbation is found intense near $z=0$ where the base flow is faster than the other spanwise locations (figure $8 a$ ). On the other hand, the spanwise velocity perturbation appears near the regions where both transverse and spanwise shear of the base flow becomes the largest ( $\left.y= \pm 1, z \simeq \pm \lambda_{z} / 4\right)$ although its strength is fairly weak compared to the transverse one (figure $8 b$ ). With the increase of $\lambda_{z}(=6)$, the region showing the largest transverse 
(a)

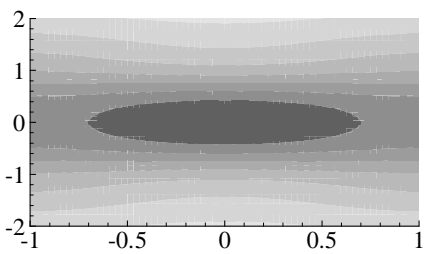

(c)

$y$

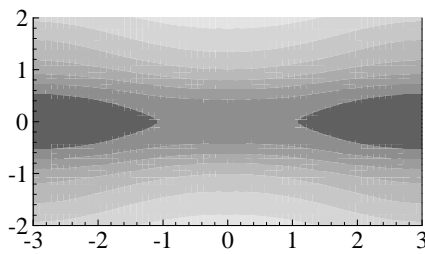

$(e)$

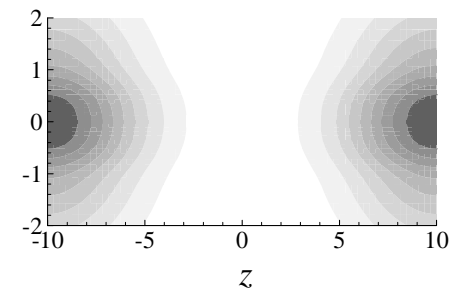

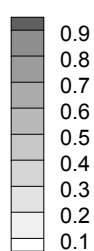

(b)

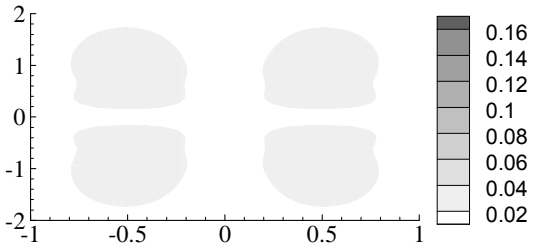

$(d)$

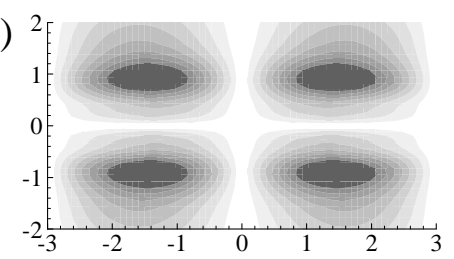

$(f)$

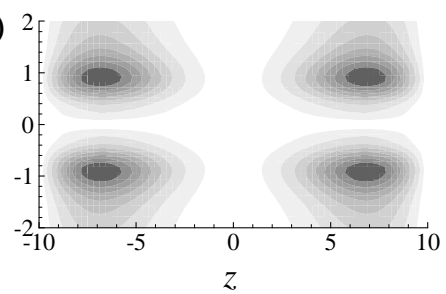

FiguRE 8. Cross-streamwise view of eigenfunctions of fundamental-mode absolute instability $\left(\mu_{0}=1\right)$ for varicose base-flow modification $(a=1.5, A=0.3$, and $R e=50):(a, b) \lambda_{z}=2$, $(c, d) \lambda_{z}=6$, and $(e, f) \lambda_{z}=20$. Here, $(a, c, e)|v| /|v|_{\max }$ and $(b, d, f)|w| /|v|_{\max }$. The velocities of the base flow in the regions near $z=0$ and $z= \pm \lambda_{z} / 2$ are respectively faster and slower than its spanwise average.

(a)

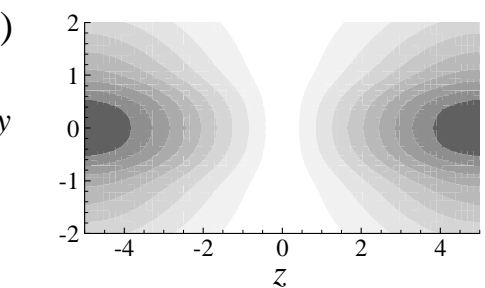

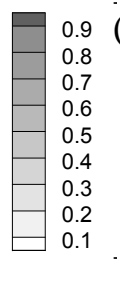

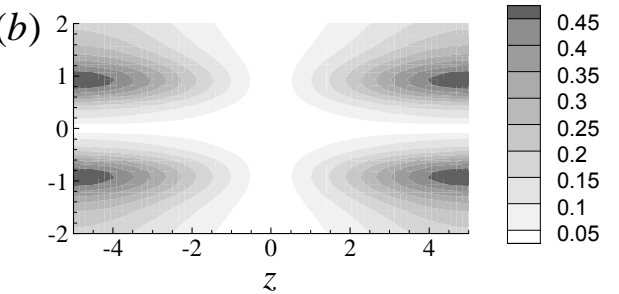

Figure 9. Cross-streamwise view of eigenfunction of subharmonic-mode absolute instability $\left(\mu_{0}=-1\right)$ for varicose base-flow modification $\left(a=1.5, A=0.3, R e=50\right.$, and $\left.\lambda_{z}=10\right)$ : (a) $|v| /|v|_{\max } ;(b)|w| /|v|_{\max }$. The velocities of the base flow in the regions near $z=0$ and $z= \pm \lambda_{z} / 2$ are respectively faster and slower than its spanwise average.

velocity perturbation is spontaneously changed to $z= \pm \lambda_{z} / 2$ at which the base flow is slower than other spanwise locations (figure $8 c$ ). The strength of the spanwise velocity perturbation relative to that of the transverse one also becomes considerably pronounced (figure $8 d$ ). For very large $\lambda_{z}(=20)$ (figure $8 e$ ), the transverse velocity perturbation near $z=0$ is negligibly small and observed only near $z= \pm \lambda_{z} / 2$ (figure 8e). The strength of the spanwise velocity perturbation is seen to be a little attenuated compared to that for $\lambda_{z}=6 h$ (figure $8 f$ ). We note that these features remain essentially the same even at larger $\lambda_{z}(=30)$.

The eigenfunction of the subharmonic-mode absolute instability $\left(\mu_{0}=-1\right)$ for varicose base-flow modification is also shown in figure 9 . Here, we only show the eigenfunction 
(a)

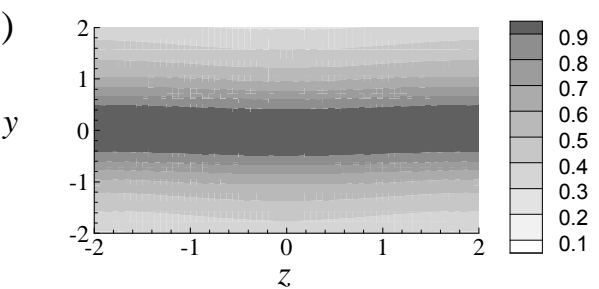

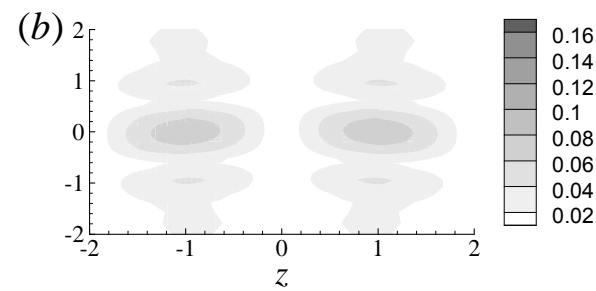

FIgURE 10. Cross-streamwise view of eigenfunction of fundamental-mode absolute instability $\left(\mu_{0}=1\right)$ for sinuous base-flow modification $\left(a=1.5, A=0.3, R e=50\right.$, and $\left.\lambda_{z}=4\right)$ : $(a)$ $|v| /|v|_{\max } ;(b)|w| /|v|_{\max }$. For $y>0$, the velocities of the base flow in the regions near $z=0$ and $z= \pm \lambda_{z} / 2$ are respectively faster and slower than its spanwise average.

for a $\lambda_{z}$ at which the subharmonic mode becomes more unstable than the fundamental mode. The spatial distribution of the transverse-velocity perturbation strength of the subharmonic mode is not very different from that of the fundamental one. However, the spanwise velocity perturbation shows an important difference: its spanwise extrema are located at $z= \pm \lambda_{z} / 2$ where the base-flow velocity is slower than other spanwise locations.

Finally, the eigenfunction for sinuous base-flow modification is shown in Fig. 10. Only the fundamental mode $\left(\mu_{0}=1\right)$ is shown in this case, as it is more unstable than the subharmonic one (see also section 3.2). Contrary to varicose modification, in this case, the transverse velocity perturbation is fairly uniformly distributed along the spanwise direction (figure 10a). The strength of the spanwise velocity perturbation relative to the transverse one is also much weaker than that for varicose modification (figure 10b), implying that the eigenfunction for the base flow with sinuous modification is not very different from that for the unmodified one. This suggests that the sinuous modification is much less effective than the varicose one in distorting the nature of two-dimensional wake instability. This is also consistent with the behavior of the growth rate shown in figure 6 .

\subsection{Effect of shear-layer thickness of base flow}

Effect of the shear-layer thickness of unmodified base flow $U_{0}(y)$ is also studied for both varicose and sinuous base-flow modifications. To quantify the amount of stabilization by the base-flow modification, we introduce the change of absolute growth rate by the given base-flow modification:

$$
\Delta \omega_{0, i}=\omega_{0, i}^{2 D}-\omega_{0, i}^{\bmod }
$$

where $\omega_{0, i}^{2 D}$ and $\omega_{0, i}^{\text {mod }}$ are absolute growth rates for the given unmodified base flow $U_{0}(y)$ and the modified base flow $U(y, z)$ respectively.

Figure $11(a)$ shows $\Delta \omega_{0, i}$ with respect to the spanwise wavelength of varicose base-flow modification for a few shear-layer thickness parameter $a$ 's of the wake profile $U_{0}(y)$ (see equation (2.14). When the shear-layer thickness is large (i.e. small $a$ ), varicose modification is quite effective in wide rages of $\lambda_{z}$. However, as the shear-layer thickness becomes thinner (i.e. large $a$ ), the stabilizing effect by varicose modification diminishes. For the thinnest shear-layer thickness considered $(a=4)$, varicose modification only slightly stabilizes absolute instability in a fairly short range of $\lambda_{z}(<5)$. The shear-layer thickness of the unmodified base flow is also found to affect the competition between the fundamental and subharmonic modes. Figure $11(b)$ shows the spanwise absolute wavenumber for the most unstable modes. As the shear layer is thinner, the spanwise wavelengths, at which 

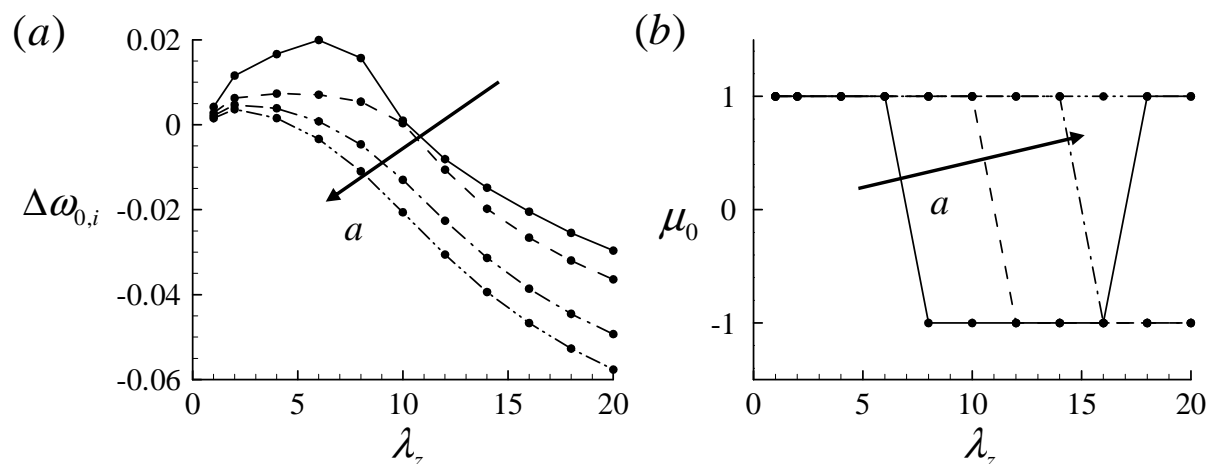

FIGURE 11. Effect of the shear-layer thickness parameter $a$ on $(a)$ the change of absolute growth rate $\Delta \omega_{0, i}$ by the base-flow modification and $(b)$ the corresponding spanwise absolute wavenumber $\mu_{0}$ for varicose modification $(A=0.2$ and $R e=50):-, a=1 ;----, a=2 ;-\cdot-\cdot-$ $a=3 ;-\cdots-\cdots-, a=4$. In (b), for $a=4, \mu_{0}=1$ for the given range of $\lambda_{z}$.

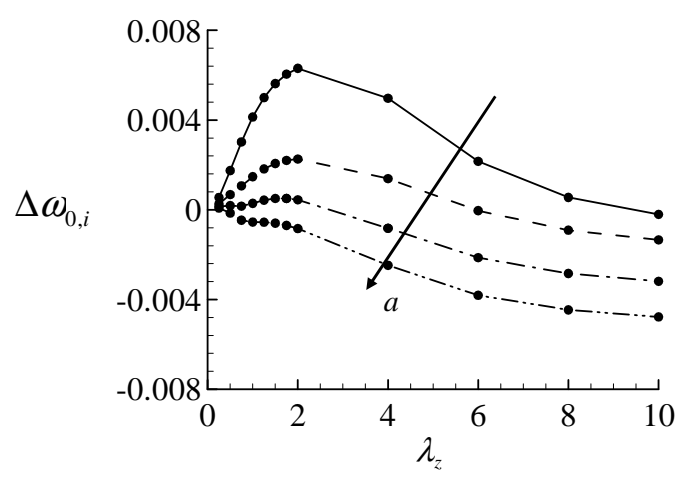

FiguRE 12. Effect of the shear-layer thickness parameter $a$ on deviation of absolute growth rate $\Delta \omega_{0, i}$ for sinuous base-flow modification $(A=0.2$ and $R e=50):-, a=1 ;----, a=2$; $-\cdot-\cdot, a=3 ;-\cdots-\cdots-, a=4$. Here, $\mu_{0}=1$ for all the cases.

the switch of the most unstable mode appears, tend to be longer. This suggests that the two modes may be competitive only for base flows with relatively thick shear layer.

The change of absolute growth rate by base-flow modification is also reported for sinuous case. Figure 12 reports $\Delta \omega_{0, i}$ with the spanwise wavelength of sinuous modification for a few shear-layer thicknesses. As in varicose modification, the shear-layer thickness significantly affects the stabilizing effect by sinuous base-flow modification. With the decrease of the shear-layer thickness, the sinuous modification becomes ineffective for stabilizing the flow. In particular, for $a=4$, the range of the spanwise wavlength which stabilize the flow does not seem to exist.

The results from both figures 11 and 12 suggest that the stabilizing effect by the spanwise waviness in base flow may diminish with the increase of shear-layer thickness of the wake. In particular, for varicose base-flow modification the $\lambda_{z}$ giving the maximum stabilization becomes shorter with the decrease of the shear-layer thickness, implying that the optimal $\lambda_{z}$ for stabilization may be correlated with the shear-layer thickness.

\subsection{Effect of the Reynolds number}

Finally, the effect of the Reynolds number is studied for both varicose and sinuous baseflow modifications. Figure $13(a)$ shows the effect of the Reynolds number on the change 

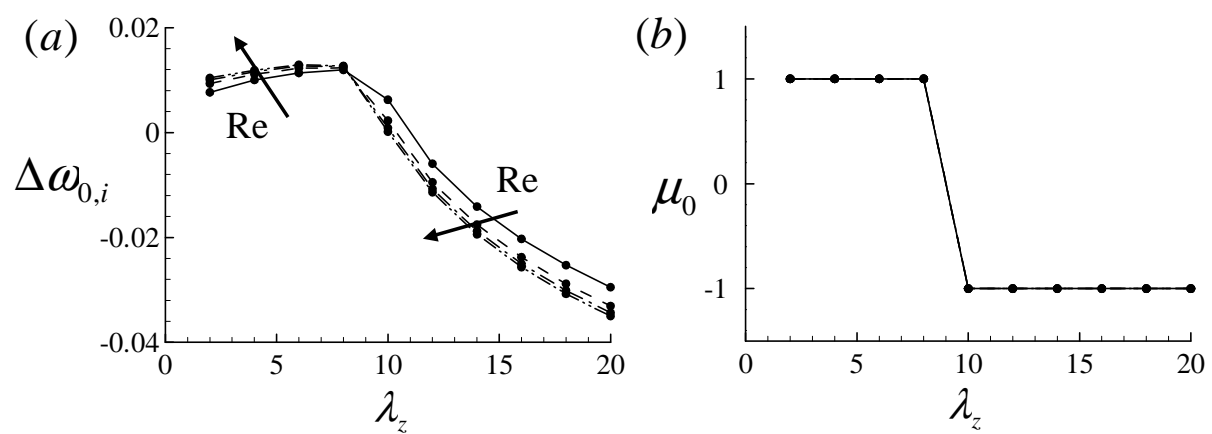

FIGURE 13. Effect of the Reynolds number on (a) deviation of absolute growth rate $\Delta \omega_{0, i}$ and $(b)$ the corresponding spanwise absolute wavenumber $\mu_{0}$ for varicose base-flow modification $(a=1.5$ and $A=0.2):-, R e=50 ;-\cdots, R e=100 ;-\cdot-\cdot, R e=150 ;-\cdots-\cdots-, R e=200$.
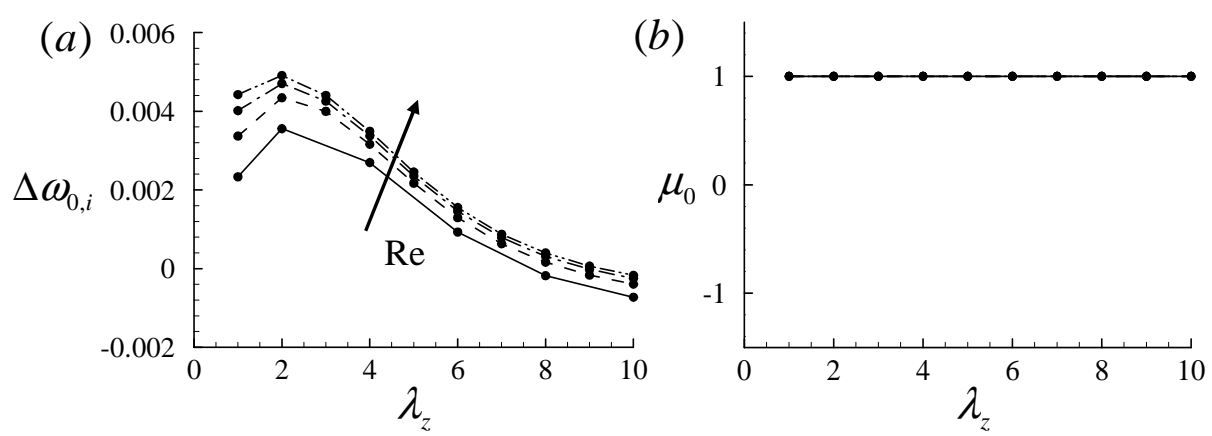

FIGURE 14. Effect of the Reynolds number on $(a)$ deviation of absolute growth rate $\Delta \omega_{0, i}$ and $(b)$ the corresponding spanwise absolute wavenumber $\mu_{0}$ for sinuous base-flow modification $(a=1.5$ and $A=0.2):-, R e=50 ;-\cdots, R e=100 ;-\cdot-\cdot, R e=150 ;-\cdots-\cdots-, R e=200$.

of absolute growth rate $\Delta \omega_{0, i}$ for varicose base-flow modification. For relatively small $\lambda_{z}(<8)$, the stabilizing effect becomes slightly more prominent with the increase of the Reynolds number. On the contrary, for large $\lambda_{z}(>10)$, the increase of the Reynolds number results in the decrease of $\Delta \omega_{0, i}$, implying that the destabilizing effect by the baseflow modification becomes enhanced by the increase of the Reynolds number. However, it is seen that the effect of the Reynolds number on the stabilization is not as critical as that of the shear-layer thickness. Furthermore, as shown in figure 13, it rarely affects the competition dynamics between the fundamental and subharmonic modes. For sinuous base-flow modification, the effect of the Reynolds number is presented in figure 14. The increase of the Reynolds number is seen to enhance the stabilizing effect by the baseflow modification at least for the spanwise wavelengths considered (figure 14a). As in varicose base-flow modification, the competition dynamics between the fundamental and subharmonic modes is found to be rarely influenced by the Reynolds number (figure 14b).

\section{Discussion}

Thus far, we have shown how the spanwise waviness in base flow influences the absolute instability in two-dimensional wake. The most important finding in the present study is probably that the spanwise wavy base-flow modifications attenuate the absolute instability when they are introduced with a relevant range of the spanwise waviness 


\begin{tabular}{lcccc}
\hline & & & & \\
Geometry & Control method & $R e$ & $\lambda_{z, \text { opt }}$ & References \\
Model vehicle & Wavy trailing edge & 40000 & $3-5 D$ & Tombazis \& Bearman (1997) \\
Rectangular cyl. & Wavy stagnation face & 40000 & $3-5 D$ & Bearman \& Owen (1998) \\
Square cyl. & Wavy stagnation face & $10-150$ & $5-6 D$ & Darekar \& Sherwin (2001) \\
Circular cyl. & blowing/suction & $40-3900$ & $4-5 D$ & Kim \& Choi (2005) \\
Model vehicle & $\quad$ Small tabs & $320-80000$ & $1.5-2 D$ & Park et al. $(2006)$
\end{tabular}

TABLE 1 . The spanwise wavelength most efficiently stabilizing vortex shedding in three-dimensional controls. Here, $R e=U_{\infty} D / \nu, U_{\infty}$ the free-stream velocity, $D$ is the maximum height of the given bluff body.

(figure 6). In many three-dimensional controls, the spanwise wavy control inputs either by passive (Tombazis \& Bearman 1997; Darekar \& Sherwin 2001; Park et al. 2006) or active (Kim et al. 2004; Kim \& Choi 2005) means strongly affect the near-wake region, leading to a non-negligible amount of modification of the near-wake base flow. What the present finding implies is that such base-flow modification results in stabilization of the near-wake absolute instabilities, which act as a wavemaker for both linear and nonlinear global instabilities (Chomaz 2005; Giannetti \& Luchini 2007; Hwang \& Choi 2008). The stabilized near-wake region is therefore likely leading to attenuation of wake global instabilities. This scenario, in a viewpoint of theory of hydrodynamic stability, explains why many three-dimensional controls are successful in regulating vortex shedding.

\subsection{Comparison with laboratory and numerical experiments}

In the present study, we have examined two types of base-flow modifications: varicose and sinuous modifications. The varicose modification models the cases in which the control input leads to the spanwise wavy deformation of the given base flows without any phase difference between its upper and lower parts. The relevant cases therefore include the controls using wavy trailing edge (Tombazis \& Bearman 1997), wavy stagnation face (Darekar \& Sherwin 2001), in-phase forcing of spanwise sinusoidal blowing and suction (Kim et al. 2004; Kim \& Choi 2005), and small tabs with symmetric configuration (Park et al. 2006). On the other hand, the sinuous modification mimics the situation where the phase difference between the upper and lower parts of the modified base flow is $\pi$. This case is comparable with the controls using out-of-phase forcing of spanwise blowing and suction (Kim et al. 2004; Kim \& Choi 2005) and staggered configuration of small tabs (Park et al. 2006).

It has been shown that the three-dimensional controls inducing varicose base-flow modification are much more effective and efficient than those leading to sinuous modification (Kim et al. 2004; Kim \& Choi 2005; Park et al. 2006). In the present study, varicose baseflow modification is found to much more effectively stabilize absolute instability than the sinuous one, consistent with these previous observations. Furthermore, the controls leading to varicose base-flow modification often result in significant spanwise distortion of the vortical structures, while those inducing sinuous modification show only little influence on them (Kim \& Choi 2005). In particular, for sufficiently large $\lambda_{z}$, the vortical structures by the controls yielding varicose base-flow modification exhibits intense fluctuation of the wall-normal velocity in the spanwise locations where the base (or mean) flow is slower than the other spanwise locations (e.g. the near-wake region behind the blowing location in Kim \& Choi 2005). These behaviors are also found in the structures of eigenfunction in the present linear analysis (see figures 8 and 10), consistent with the previous observation. 
The spanwise wavelengths providing stabilization of absolute instability are also in good agreement with those stabilizing vortex shedding. Table 1 summarizes the spanwise wavelength which have been found to most effectively stabilize vortex shedding in previous laboratory and numerical experiments. We note that only the cases resulting in varicose base-flow modification are presented due to the lack of data in the cases for sinuous base-flow modification. Although the reported optimal spanwise wavelength a little deviate depending on the geometry, control method, and the Reynolds number, it firmly stays in the range of $\lambda_{z, \text { opt }} \simeq 1-6 D$. In the present study, the varicose base-modification is found to stabilize absolute instability for $\lambda_{z} \simeq 1-10$ though the range of the spanwise wavelength depends a little on the amplitude of modification (see figure $6 a$ ). Since the length scale $h$ defined in the present study is the half width of the wake, it would be reasonable to assume $D \simeq 2 h$. This implies that the optimal spanwise wavelength reported in previous laboratory and numerical experiments would be about $\lambda_{z} \simeq 2-12$, showing good agreement with the present linear analysis.

In spite of such good agreement, care should be taken in interpreting the present results. First, although the base flow in the present study is chosen from a numerical simulation result at low Reynolds numbers (Kim 2005), it is still a 'minimal' representation of real base flows modified by the spanwise varying control input. For example, describing the base flow with (2.13) may be reasonable if the input of the designed three-dimensional control is purely sinusoidal and its amplitude is small enough. However, in many cases, the control input is neither purely sinusoidal nor very small. Therefore, the base flows in real cases are likely being much more complicated than that given by (2.13): for example, the spanwise uniformity of the base flow could be deformed by the given control input, and the base flow may contain multiple spanwise wave components. Therefore, real base flows obtained by either numerical simulation or experiments should be studied by more precise analysis, but this is beyond the scope of the present study.

Second, the present analysis is linear, thus it is strictly valid only for small-size perturbation. This is particularly important for large $\lambda_{z}$, at which both the varicose and sinuous base-flow modifications are found to destabilize absolute instability. However, it is not clear whether such destabilization would enhance strength of the resulting vortex shedding. In fact, when three-dimensional control is applied with sufficiently long spanwise wavelength, vortex shedding often exhibits multiple frequencies interacting among themselves instead of the enhancement in its strength (Darekar \& Sherwin 2001; Kim \& Choi 2005).

Finally, the present analysis assumes that base flow is parallel, but it is often strongly non-parallel in real cases. This difference may be important particularly in interpreting the appearance of subharmonic mode. We note that the subharmonic mode appears only for the sufficiently large amplitude of varicose base-flow modification (see figure $7 b$ ) and that it is strongly damped for the small amplitude of the modification. This implies that, in real flows, the absolute instability associated with the subharmonic mode would be only active in the near-wake region where the base flow is strongly distorted by the given spanwise waviness, while it may be strongly damped in further downstream where the effect of the control input is expected to diffuse out. As a result, the absolute growth rate of the subharmonic mode would experience stronger non-parallelism along the streamwise direction than that of the fundamental mode. This suggests that the global instability from the subharmonic absolute mode may be more stable than that from the fundamental mode. 


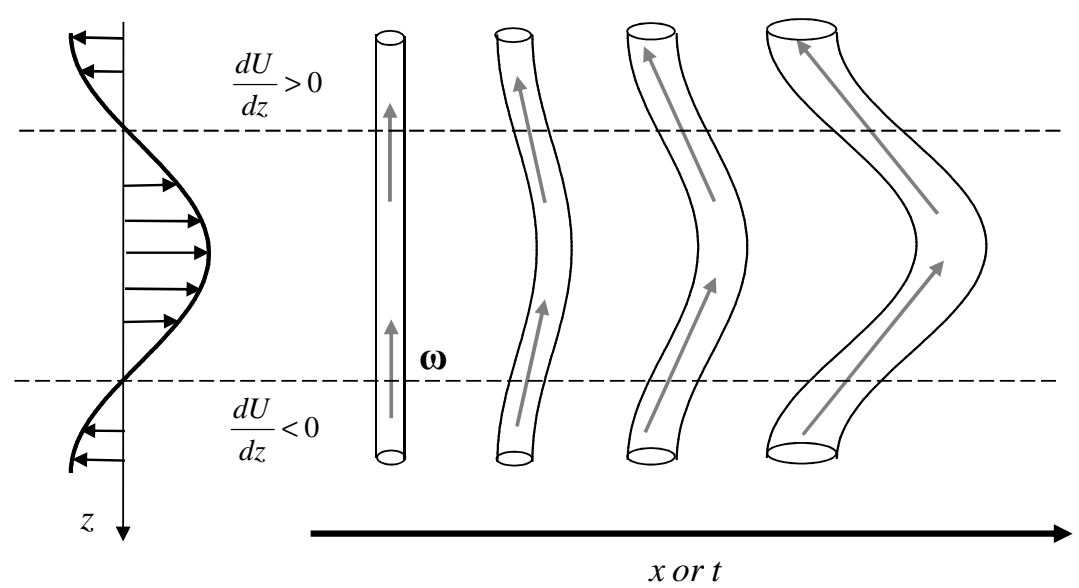

FiguRE 15. Evolution of a spanwise vortex obeying dynamics described by (4.2). Here, thickness of the vortex tube represents its strength.

\subsection{Physical mechanism of stabilization}

In this section, we discuss physical mechanism of stabilization of the two-dimensional wake instability by analyzing vortex dynamics. Taking curl of (2.12) gives the following equation for vorticity perturbations:

$$
\begin{gathered}
\frac{\partial \omega_{x}}{\partial t}+U \frac{\partial \omega_{x}}{\partial x}=\frac{\partial v}{\partial x} \frac{\partial U}{\partial z} \\
\frac{\partial \omega_{z}}{\partial t}+U \frac{\partial \omega_{z}}{\partial x}+v \frac{\partial \Omega_{z}}{\partial y}+w \frac{\partial \Omega_{z}}{\partial z}=\frac{\partial w}{\partial z} \Omega_{z}+\frac{\partial w}{\partial y} \frac{\partial U}{\partial z}
\end{gathered}
$$

where $\omega_{x}$ and $\omega_{z}$ are respectively the streamwise and spanwise vorticity perturbations, and $\Omega_{z}=-d U / d y$ is the background spanwise vorticity given by the base flow. Here, we note that all the terms associated with the transverse vorticity fluctuation are neglected as the computed eigenfunctions exhibit negligibly small transverse vorticity perturbation. The terms in the left-hand side of (4.1a) represent the advection of $\omega_{x}$ by the base flow, and the term in its right-hand side describes the tilting of $\omega_{z}$ by the spanwise base-flow shear. In $(4.1 b)$, the first two terms in the left-hand side are the advection of $\omega_{z}$ by the base flow, while the third and fourth terms respectively represent the production of $\omega_{z}$ by the transverse and spanwise base-flow shear. In particular, the third term appears as a source term of the two-dimensional inflectional instabilities. The two terms in the righthand side of $(4.1 b)$ represents the stretching of the base-flow vorticity and the tilting of $\omega_{x}$ by the spanwise base-flow shear.

We first consider sufficiently large spanwise wavelength $\lambda_{z}$ for the base-flow modification. Since $\partial U / \partial z \sim 1 / \lambda_{z}$, this gives negligibly small contribution to all the terms involving the spanwise gradient of base flows: two vorticity tilting terms respectively in $(4.1 a)$ and $(4.1 b)$, and the production term by spanwise base-flow shear (the fourth term in the left-hand side of $(4.1 b)$ ). In this case, the flow is found only destabilized (figure 6), implying that these terms are likely playing a crucial role in stabilizing absolute instability. Furthermore, as the shear-layer thickness becomes thinner (i.e. amplitude of $\partial U / \partial y$ becomes larger at the shear layer), the stabilizing effect also diminishes (see figures 11 and 12). This suggests that the production term by spanwise base-flow shear is not likely involved in stabilization either. From these observations, the vortex dynamics associated 

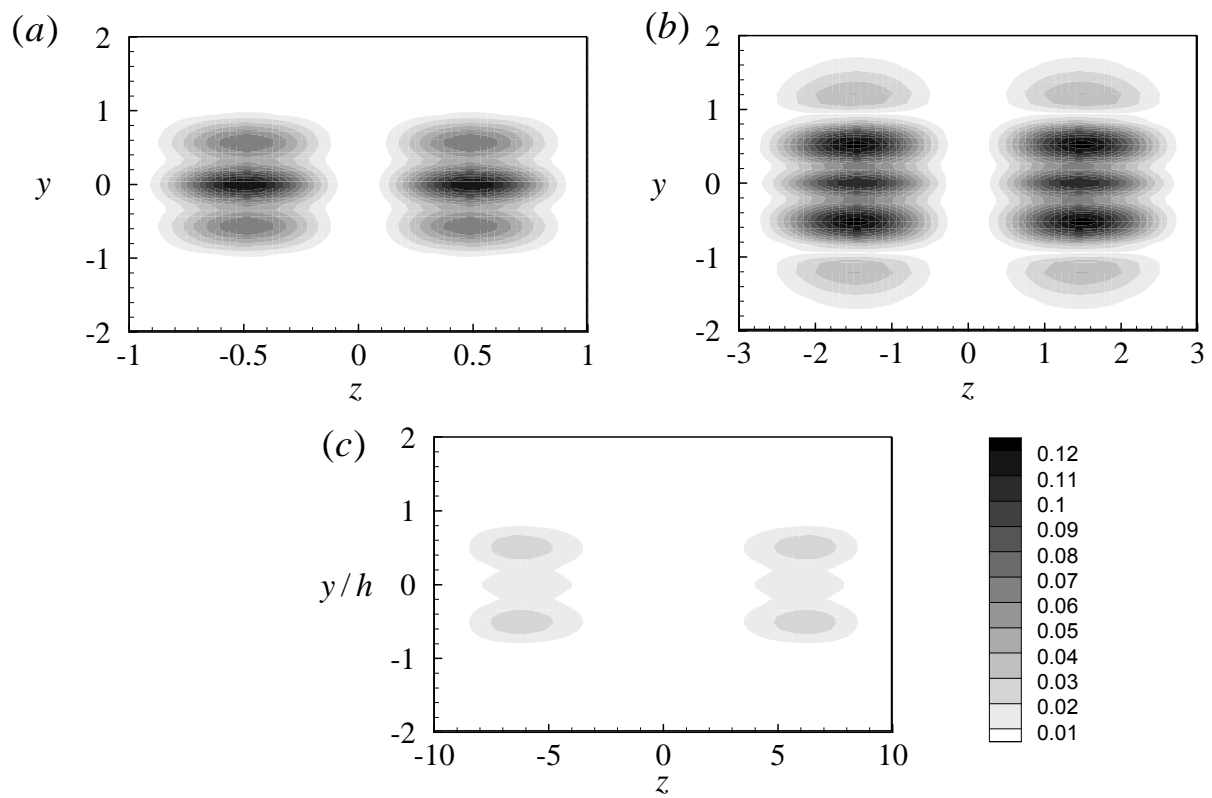

FIGURE 16. Cross-streamwise view of $|\partial w / \partial y \partial U / \partial z|$ normalized by $|v|_{\text {max }}$ for varicose base-flow modification: (a) $\lambda_{z}=2 ;(b) \lambda_{z}=6 ;(c) \lambda_{z}=20$.

with stabilization may be described by the following equations:

$$
\begin{aligned}
\frac{D \omega_{x}}{D t} & \sim \omega_{z} S_{x z}, \\
\frac{D \omega_{z}}{D t} & \sim \omega_{x} S_{x z},
\end{aligned}
$$

where $S_{x z}$ is the deformation-rate tensor originating from the spanwise shear of the base flow.

To gain a physical insight into the vortex dynamics described by (4.2), we consider an evolution of a spanwise vortex tube as illustrated in figure 15 . We set the spanwise vortex tube such that $\omega_{z}<0$ (the first left vortex in figure 15). Equation (4.2a) suggests that the vortex tube is tilted downstream with generation of positive $\omega_{x}$ in the region where $d U / d z\left(=S_{x z}\right)<0$. Similarly, in the region where $d U / d z\left(=S_{x z}\right)>0$, the vorticity vector is tilted upstream. This implies that the given spanwise vortex gradually evolves into a $\Lambda$-shape vortex with a loop at the spanwise location where the base flow is faster than its spanwise average. This explains the appearance of the $\Lambda$-shape vortices observed in most of previous experimental and numerical studies (Darekar \& Sherwin 2001; Kim et al. 2004; Kim \& Choi 2005; Park et al. 2006), and the location of its loop is consistent with these studies (see e.g. figure $12 c$ in Kim \& Choi 2005). However, it should be pointed out that the dynamics of single spanwise vortex tube exhibits the strengthening rather than the attenuation with generation of $\omega_{x}$ : in both the regions of $d U / d z<0$ and $d U / d z>0, D \omega_{z} / D t\left(=\omega_{x} S_{x z}\right)<0$ for $\omega_{z}<0$ (see $\left.(4.2 b)\right)$. Therefore, the appearance of $\Lambda$-shape vortices only itself does not explain the physical mechanism of stabilization of the instability, of which the spatial structure constitutes an array of counter-rotating spanwise vortices.

From these observations, the term $\omega_{x} S_{x z}$ in $(4.2 b)$, which probably acts differently 
with the array of alternating $\Lambda$-vortices from that with the single $\Lambda$-vortex, turns out to be the only term related to physical mechanism of the stabilization. Importantly, the eigenfunctions in the present analysis show that this term is strongly correlated to the spanwise wavelength providing stabilization of absolute instability. In figure 16, we visualize the intensity of the tilting term $\omega_{x} S_{x z}\left(=\partial_{y} w \partial_{z} U\right)$ for the fundamental absolute mode. For $\lambda_{z}=2$ and $\lambda_{z}=6$, this tilting term shows very large value along the centerline of the wake (figures $16 a$ and $b$ ). In particular, the term for $\lambda_{z}=6$, which provides the maximum stabilization of absolute instability, is found even more intense than that for $\lambda_{z}=2$. On the contrary, for $\lambda_{z}=20$, this term becomes very weak due to the small spanwise shear in base flow (figure 16c). This finding suggests that the tilting of the streamwise vortices by spanwise shear is probably directly involved in attenuation of instability and/or vortex shedding.

Taking all these together, the physical mechanism of stabilization constitutes two key processes: 1) formation of streamwise vortices from tilting of the two-dimensional spanwise Kármán vortices by spanwise base-flow shear $((4.2 a)) ; 2)$ interaction of the array of the counter-rotating spanwise vortices with tilting of the formed streamwise vortices by the spanwise base-flow shear $((4.2 b))$. Since the present analysis mainly relies on the long-time asymptotic response of the given linear system, it is difficult to proceed further analysis on transient vortex dynamics. However, it should be pointed out that the interaction among $\Lambda$-shape counter-rotating vortices has been observed as an essential process for stabilization of vortex shedding. For example, Kim \& Choi (2005) and Park et al. (2006) observed that the controlled flow field exhibits different oscillation phase depending on the spanwise location, which is likely a consequence of the interaction among the counter-rotating $\Lambda$-shape vortices.

\subsection{Sensitivity of absolute instability to spanwise wavy base-flow modification}

Three-dimensional controls have often been found to be much more efficient and effective than two-dimensional ones in stabilizing wake instability and vortex shedding (Kim et al. 2004; Kim \& Choi 2005; Park et al. 2006). There are two possible origin of such effectiveness and efficiency. First, the base (or mean) flow of two-dimensional wake might be very sensitively deformed by a small amount of control input, and this subsequently results in such a very efficient stabilization of wake instability. The second scenario is that the wake instability itself might be highly sensitive to spanwise periodic base-flow modification. We note that the present analysis, which studies linear instability of the 'prescribed' base flow, enables us to examine the latter scenario. This section is therefore aimed at examining whether modifying base flow with a given spanwise periodicity yields highly sensitive change of absolute instability.

We first consider the normal-mode solution of (2.12) in the primitive variable form: $\mathbf{u}(x, y, z)=\hat{\mathbf{q}}(y, z) e^{i(\alpha x+\beta z-\omega t)}$, where $\mathbf{u}=\left[\begin{array}{llll}u & v & w & p\end{array}\right]^{T}$ and $\hat{\mathbf{q}}=\left[\begin{array}{llll}\hat{q}_{u} & \hat{q}_{v} & \hat{q}_{w} & \hat{q}_{p}\end{array}\right]^{T}$ is a $z-$ periodic vector function. This gives the following eigenvalue problem leading to dispersion relation of (2.12):

$$
-i \omega \mathcal{H} \hat{\mathbf{q}}=\mathcal{L}_{2 D}\left(\alpha,-i \frac{\partial}{\partial z}+\beta ; U_{0}\right) \hat{\mathbf{q}}+A \mathcal{L}_{3 D}(\alpha ; \Delta U) \hat{\mathbf{q}},
$$

where the operators $\mathcal{H}, \mathcal{L}_{2 D}$, and $\mathcal{L}_{3 D}$ are given in Appendix B.

Now, we assume that the amplitude of the base-flow modification is small enough $(A \ll 1)$. In this case, the adjoint-based sensitivity analysis (e.g. Hwang \& Choi 2006) provides a way to compute the leading-order variation of complex absolute frequency by a small amount of the base-flow modification. The complex absolute frequency and the eigenmode of (4.3) are written using regular asymptotic expansion around the complex 


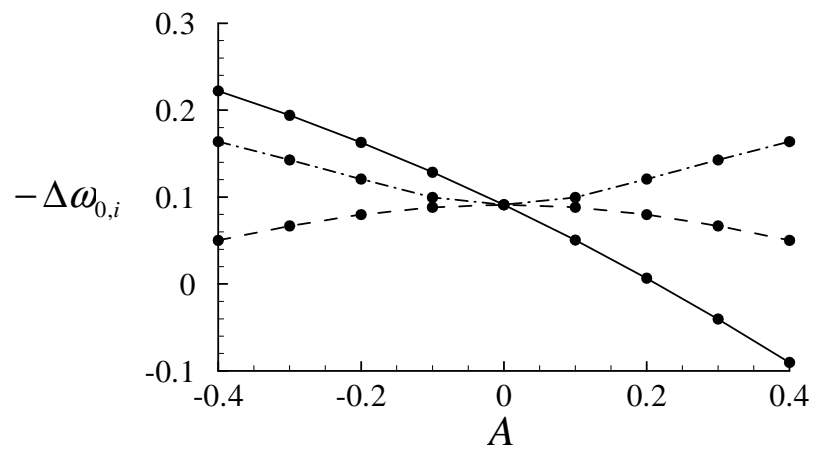

FiguRE 17. Comparison of change of the absolute growth rate $\Delta \omega_{0, i}$ between two-dimensional $\left(U(y)=U_{0}(y)+A U_{1}(y)\right)$ and three-dimensional $\left(U(y)=U_{0}(y)+A U_{1}(y) \cos \left(2 \pi / \lambda_{z} y\right)\right)$ base-flow modifications $(a=1.5$ and $R e=50)$ : - , two-dimensional modification; - - - - , three-dimensional varicose modification with $\lambda_{z}=6 ;-\cdot-\cdot-$, three-dimensional varicose modification with $\lambda_{z}=20$.

absolute frequency $\left(\omega_{0}^{2 D}\right)$ and the eigenmode $\left(\mathbf{q}_{0}^{2 D}\right)$ for two-dimensional base flow $U_{0}$ :

$$
\begin{gathered}
\omega_{0}=\omega_{0}^{2 D}+A \delta \omega_{0}+O\left(A^{2}\right), \\
\hat{\mathbf{q}}_{0}=\hat{\mathbf{q}}_{0}^{2 D}(y)+A \delta \hat{\mathbf{q}}_{0}(y, z)+O\left(A^{2}\right),
\end{gathered}
$$

where $\delta \omega_{0}$ and $\delta \hat{\mathbf{q}}_{0}(y)$ are the leading-order variations of the complex absolute frequency and the eigenmode respectively. We introduce a standard inner product $\langle\hat{\mathbf{f}}, \hat{\mathbf{g}}\rangle \equiv$ $1 / \lambda_{z} \int_{0}^{\lambda_{z}} \int_{-\infty}^{\infty} \hat{\mathbf{f}}^{H} \mathcal{H} \hat{\mathbf{g}} d y d z$ where the superscript ${ }^{H}$ indicates the complex conjugate transpose, and $\hat{\mathbf{f}}$ and $\hat{\mathrm{g}}$ are arbitrary vector functions defined in $x$ and $z$ space. Then, the solvability condition gives the following leading-order variation of the complex absolute frequency:

$$
\delta \omega_{0}=\frac{\left\langle i \mathcal{L}_{3 D}\left(\alpha_{0} ; \Delta U\right) \hat{\mathbf{q}}_{0}^{2 D}, \hat{\mathbf{p}}_{0}^{2 D}\right\rangle}{\left\langle\hat{\mathbf{q}}_{0}^{2 D}, \hat{\mathbf{p}}_{0}^{2 D}\right\rangle},
$$

where $\hat{\mathbf{p}}_{0}^{2 D}$ is the adjoint absolute mode from the adjoint operator of $\mathcal{L}_{2 D}$ with respect to the given inner product. We note that $\delta \omega_{0}$ is not affected by the change of absolute wavenumbers $\alpha_{0}$ and $\beta_{0}$ due to the condition (2.10) (Hwang \& Choi 2006).

It is interesting to observe that $\mathcal{L}_{3 D}\left(\alpha_{0} ; \Delta U\right)$ in (4.5) does not contain any spanwise uniform components (see also Appendix B). This results in $\delta \omega_{0}=0$, implying that modifying the given base flow only sinusoidally along the spanwise direction does not give any change in the absolute frequency $\omega_{0}$ at the leading order. In sharp contrast, two-dimensional base-flow modification yields the leading-order change in the absolute frequency (Hwang \& Choi 2006). Consequently, the spanwise wavy base-flow modification gives

$$
\Delta \omega_{0}^{3 D} \sim O\left(A^{2}\right),
$$

while two-dimensional modification yields

$$
\Delta \omega_{0}^{2 D} \sim O(A)
$$

Here, $\Delta \omega_{0}^{3 D}$ and $\Delta \omega_{0}^{2 D}$ are the changes of absolute frequency, respectively, by the spanwise wavy and the two-dimensional modifications. We note that this analytical result is seen throughout the present paper. For example, the change of absolute growth rate by spanwise wavy base-flow modification is only $O\left(A^{2}\right)$ or less than that (see figures 6 and 
11-14), consistent with (4.6a). On the contrary, two-dimensional modification exhibits $O(A)$ changes in the absolute growth rate. To support these analytical findings, in figure 17 , we also compare the effect of a two-dimensional base-flow modification with that of three-dimensional varicose modification by considering the two-dimensional modification as $U(y)=U_{0}(y)+A U_{1}(y)$, where $U_{1}(y)$ is the varicose modification defined in (2.15). It is seen that the two-dimensional base-flow modification indeed yields much larger change than the spanwise wavy modification for small $A$ and that the amount of change in the absolute growth rate is proportional to $O(A)$ consistent with (4.6b). Furthermore, the three-dimensional modification results in $\Delta \omega_{0}^{3 D} \sim O\left(A^{2}\right)$ for small $A$, consistent with $(4.6 a)$.

The finding in this section suggests that absolute instability of two-dimensional wakes is much less sensitive to spanwise wavy base-flow modifications than to two-dimensional modifications. Alternatively, this implies that the high effectiveness and efficiency of several three-dimensional controls compared to the two-dimensional ones (Kim et al. 2004; Kim \& Choi 2005; Park et al. 2006) are not from sensitive response of wake instability itself to spanwise waviness of base flow, but from sensitive modification of base (or mean) flow by the given spanwise wavy control input.

\section{Concluding remarks}

Thus far, we have investigated the role of the spanwise periodic base-flow modification in absolute instability of parallel wakes to understand the mechanism by which threedimensional controls stabilize vortex shedding in two-dimensional bluff-body wakes. The main findings of the present study are summarized as follows:

(a) The spatio-temporal evolution of instabilities in the streamwise parallel and spanwise periodic base flows is studied by applying Floquet theory to linearized Navier-Stokes equation. The criterion for absolute instability of the given base flow is analytically derived with dispersion relation by extending the procedure in Brevdo \& Bridges (1996), and is numerically verified by searching the saddle points both in the complex streamwise plane and in the complex plane of eigenvalue of the monodromy operator.

(b) Two types of the spanwise periodic base-flow modification are considered: varicose and sinuous modification. The varicose base-flow modification is found to much more effective than the sinuous one, consistent with previous experimental and numerical studies where the controls yielding the varicose modification are more effective than those inducing the sinuous one. The optimal spanwise wavelengths of the varicose and sinuous modifications are about $\lambda_{z} \simeq 6-10$ and $\lambda_{z} \simeq 2$, respectively, and the one for the varicose modification compares well with the spanwise length of three-dimensional controls that provide the maximum stabilization of vortex shedding. For both the modifications, the optimal spanwise wavelengths slightly depend on the amplitude of modification, and they are highly correlated to shear-layer thickness of the given base flow.

(c) The physical mechanism of stabilization is shown to involve two key processes: 1) formation of streamwise vortices from tilting of the two-dimensional spanwise Kármán vortices by spanwise base-flow shear; 2) interaction of the tilted Kármán vortex street with tilting of the streamwise vortices by the spanwise base-flow shear. This finding is consistent with previous observations where the interaction between bent Kármán vortices is shown to be an essential process for stabilization of vortex shedding (e.g. Kim et al. 2004; Kim \& Choi 2005; Park et al. 2006).

(d) The sensitivity of absolute instability to spanwise wavy base-flow modification is finally studied. It is shown that the change of complex absolute frequency by spanwise wavy base-flow modification is proportional to the square of the modification ampli- 
tude, whereas that by two-dimensional base-flow modification is found proportional to the modification amplitude itself. This finding suggests that absolute instability in twodimensional wakes is much less sensitive to spanwise wavy base-flow modification than to two-dimensional modification. This suggests that the high efficiency of several threedimensional controls (e.g. Kim et al. 2004; Kim \& Choi 2005; Park et al. 2006) is probably because the base (or mean) flow is highly distorted by the given control inputs.

The present study has shown the applicability of Floquet theory for understanding spatio-temporal dynamics (absolute and convective nature) of instabilities in linearized Navier-Stokes system with spatially periodic base flows using a given numerical eigenvalue solver. It is certainly fortunate that the governing equation considered here does not have any spanwise biased base-flow advection, and this greatly simplifies the searching process of the spanwise absolute wavenumber. However, it should also be pointed out that this kind of linearized system is often encountered in many interesting physical systems: for example, instability of streaks in wall-bounded flows (Waleffe 1995; Reddy et al. 1998; Schoppa \& Hussain 2002; Brandt et al. 2003; Park et al. 2011). Therefore, the present analysis would provide a useful technical framework in analyzing absolute and convective nature of the given instabilities in Navier-Stokes system.

Probably, the most important finding in the present study is that the spanwise waviness in base flow of two-dimensional wake results in stabilization of absolute instability. Since the near-wake region, where the local base-flow profile exhibits absolutely unstable nature, has been understood as a wavemaker for both linear and nonlinear global instabilities (Chomaz 2005; Giannetti \& Luchini 2007; Marquet et al. 2008; Hwang \& Choi 2008), the present study explains why many three-dimensional controls stabilize two-dimensional vortex shedding. However, it should be emphasized that absolute instability of the two-dimensional wake is found not very sensitive to the spanwise waviness in the given base flow. This therefore raises an important question to be asked in the future study: what is the mechanism of such effective base-flow modification by the control inputs in three-dimensional control?

Although the present analysis is not capable of directly answering this question, several recent studies suggests that there might be some effective base-flow modification mechanisms by the spanwise wavy control inputs. First, the spanwise wavy input may trigger a resonate response with the mode-A instability (Williamson 1996) and this may significantly distort base (or mean) flow. In fact, a similar mechanism has been suggested by Darekar \& Sherwin (2001), where they conjectured that the optimal spanwise wavelength of vortex shedding suppression is associated with the wavelength of the mode-A instability. However, it has remained not very clear why such forcing of the mode-A instability induces suppression of vortex shedding rather than destabilization. In the present study, we have shown that wake instability could be suppressed when the base flow is highly modified with a given spanwise waviness. Furthermore, the optimal spanwise wavelength providing the maximum suppression of absolute instability is found not very far from the typical spanwise wavelength of the mode-A instability $\left(\lambda_{z} \simeq 4 \sim 6 D\right.$ depending on the bluff-body geometry). This suggests that when the control input leads to strong spanwise wavy distortion of base (or mean) flow through interaction with the mode-A instability, it would stabilize wake instability instead of destabilization. However, it is not so clear whether such a resonate interaction with the mode-A instability would also be active even at high Reynolds numbers where the wake is completely turbulent (e.g. $R e_{D}>1000$ ), and we should point out that may three-dimensional controls are also quite successful at high Reynolds numbers.

It is finally interesting to note that several three-dimensional controls, which specially exhibits fairly high efficiency, are often designed to directly inject a small amount of 
transverse momentum: e.g. the spanwise blowing/suction (Kim et al. 2004; Kim \& Choi 2005) and small vertical tabs (Park et al. 2006). The large amount of base-flow modification, required for highly efficient stabilization of vortex shedding, suggests that this injected transverse momentum should experience a significant amount of amplification in separating shear layer while it is transformed into the streamwise momentum. It is interesting to note that this amplification process of the transverse momentum is reminiscent of the so-called lift-up effect (Ellingsen \& Palm 1975; Landahl 1980), which describes amplification of the streaky structures of streamwise velocity from streamwise vortical perturbations (particularly transverse velocity) under the mean shear. The lift-up effect is often represented as a vortex tilting process,

$$
\frac{D \omega_{y}}{D t} \sim \omega_{x} \frac{\partial U}{\partial y}
$$

and it has been widely understood to play a key role in formation of the streaks in bypass transition (Butler \& Farrell 1992; Reddy \& Henningson 1993; Schmid \& Henningson 2001) and fully-developed turbulent flows (Butler \& Farrell 1993; del Álamo \& Jiménez 2006; Pujals et al. 2009; Hwang \& Cossu 2010a,b). However, in wall-bounded flows, the strong amplification of the streaky motions via this mechanism is often observed at relatively high Reynolds numbers. Therefore, the relevance of this mechanism at low Reynolds numbers remains an open question as many three-dimensional controls are also often efficient enough in this case.

Y.H. is supported by the European Commission through a Marie Curie fellowship, and gratefully acknowledges to Prof. Carlo Cossu for his encouragement to continue this work. H.C. acknowledges the support from National Research Foundation, Ministry of Education, Science, and Technology, Korea (20120008740, 2012055647).

\section{Appendix A. Transformation of linearized Navier-Stokes operator for application of Floquet theory}

We consider Navier-Stokes equation linearized around the streamwise parallel and spanwise periodic base flow. We neglect the transverse and spanwise components in the base flow, resulting in $(U(y, z), 0,0)$ where $U(y, z)=U\left(y, z+\lambda_{z}\right)$ and $\lambda_{z}$ is the spanwise period. We note that this assumption does not greatly limit the present analysis as the transverse and spanwise components of the base (or mean) flow induced by threedimensional control are found much smaller than the streamwise component (Kim \& Choi 2005; Kim 2005). The linearized equations of motion are then written as follows:

$$
\begin{gathered}
\frac{\partial u}{\partial x}+\frac{\partial v}{\partial y}+\frac{\partial w}{\partial z}=0 \\
\frac{\partial u}{\partial t}+U \frac{\partial u}{\partial x}+v \frac{\partial U}{\partial y}+w \frac{\partial U}{\partial z}=-\frac{\partial p}{\partial x}+\frac{1}{R e} \nabla^{2} u \\
\frac{\partial v}{\partial t}+U \frac{\partial v}{\partial x}=-\frac{\partial p}{\partial y}+\frac{1}{R e} \nabla^{2} v \\
\frac{\partial w}{\partial t}+U \frac{\partial w}{\partial x}=-\frac{\partial p}{\partial z}+\frac{1}{R e} \nabla^{2} w
\end{gathered}
$$

where $u, v$, and $w$ are respectively the streamwise, transverse, and spanwise velocity perturbations, and $p$ is the pressure perturbation.

Here, we show that these linearized Navier-Stokes equations (A 1) are transformed 
to the form of (2.1). It is convenient to consider the transverse velocity $(v)$ and transverse vorticity $(\eta)$ form of equations (A 1) (Waleffe 1995; Reddy et al. 1998; Schmid \& Henningson 2001):

$$
\begin{aligned}
&\left(\frac{\partial}{\partial t}+U \frac{\partial}{\partial x}\right) \nabla^{2} v+\frac{\partial^{2} U}{\partial z^{2}} \frac{\partial v}{\partial x}+2 \frac{\partial U}{\partial z} \frac{\partial^{2} v}{\partial x \partial z} \\
&-\frac{\partial^{2} U}{\partial y^{2}} \frac{\partial v}{\partial x}-2 \frac{\partial U}{\partial z} \frac{\partial^{2} w}{\partial x \partial y}-2 \frac{\partial^{2} U}{\partial y \partial z} \frac{\partial w}{\partial x}=\frac{1}{R e} \nabla^{4} v \\
& \frac{\partial \eta}{\partial t}+U \frac{\partial \eta}{\partial x}-\frac{\partial U}{\partial z} \frac{\partial v}{\partial y}+v \frac{\partial^{2} U}{\partial y \partial z}+\frac{\partial U}{\partial y} \frac{\partial v}{\partial z}+w \frac{\partial^{2} U}{\partial z^{2}}=\frac{1}{R e} \nabla^{2} \eta \\
& \frac{\partial^{2} w}{\partial x \partial x}+\frac{\partial^{2} w}{\partial z \partial z}=-\frac{\partial \eta}{\partial x}-\frac{\partial^{2} v}{\partial y \partial z}
\end{aligned}
$$

Now, we introduce a state variable $\psi$ as follows:

$$
\psi(x, y, z, t)=\left[\begin{array}{llllllll}
v & v^{\prime} & v^{\prime \prime} & v^{\prime \prime \prime} & \eta & \eta^{\prime} & w & w^{\prime}
\end{array}\right]^{T},
$$

where

$$
v^{\prime} \equiv \frac{\partial v}{\partial z}, \quad v^{\prime \prime} \equiv \frac{\partial v^{\prime}}{\partial z}, \quad v^{\prime \prime \prime} \equiv \frac{\partial v^{\prime \prime}}{\partial z}, \quad w^{\prime} \equiv \frac{\partial w}{\partial z}, \quad \eta^{\prime} \equiv \frac{\partial \eta}{\partial z}
$$

Rewriting (A 2) in terms of $\psi$ gives (2.1) with the following definitions of $\mathcal{M}$ and $\mathcal{L}$ :

$$
\begin{aligned}
& \mathcal{M} \equiv\left(\begin{array}{cccccccc}
0 & 0 & 0 & 0 & 0 & 0 & 0 & 0 \\
0 & 0 & 0 & 0 & 0 & 0 & 0 & 0 \\
0 & 0 & 0 & 0 & 0 & 0 & 0 & 0 \\
-R e\left(\frac{\partial^{2}}{\partial x^{2}}+\frac{\partial^{2}}{\partial y^{2}}\right) & 0 & -R e & 0 & 0 & 0 & 0 & 0 \\
0 & 0 & 0 & 0 & 0 & 0 & 0 & 0 \\
0 & 0 & 0 & 0 & -R e & 0 & 0 & 0 \\
0 & 0 & 0 & 0 & 0 & 0 & 0 & 0 \\
0 & 0 & 0 & 0 & 0 & 0 & 0 & 0
\end{array}\right) \\
& \mathcal{L}\left(y, z ;-i \frac{\partial}{\partial x}\right) \equiv\left(\begin{array}{cccccccc}
0 & 1 & 0 & 0 & 0 & 0 & 0 & 0 \\
0 & 0 & 1 & 0 & 0 & 0 & 0 & 0 \\
0 & 0 & 0 & 1 & 0 & 0 & 0 & 0 \\
L_{v}^{v} & L_{v^{\prime}}^{v} & L_{v^{\prime \prime}}^{v} & 0 & 0 & 0 & L_{w}^{v} & 0 \\
0 & 0 & 0 & 0 & 0 & 1 & 0 & 0 \\
L_{v}^{\eta} & L_{v^{\prime}}^{\eta} & 0 & 0 & L_{\eta}^{\eta} & 0 & L_{w}^{\eta} & 0 \\
0 & 0 & 0 & 0 & 0 & 0 & 0 & 1 \\
0 & -\frac{\partial}{\partial y} & 0 & 0 & -\frac{\partial}{\partial x} & 0 & -\frac{\partial^{2}}{\partial x^{2}} & 0
\end{array}\right)
\end{aligned}
$$

where

$$
\begin{gathered}
L_{v}^{v} \equiv \operatorname{Re}\left[U \frac{\partial}{\partial x}\left(\frac{\partial^{2}}{\partial x^{2}}+\frac{\partial^{2}}{\partial y^{2}}\right)+\frac{\partial^{2} U}{\partial z^{2}} \frac{\partial}{\partial x}-\frac{\partial^{2} U}{\partial y^{2}} \frac{\partial}{\partial x}\right]-\left[\frac{\partial^{4}}{\partial y^{4}}-2 \frac{\partial^{4}}{\partial x^{2} \partial y^{2}}+\frac{\partial^{4}}{\partial x^{4}}\right], \\
L_{v^{\prime}}^{v} \equiv 2 \operatorname{Re} \frac{\partial U}{\partial z} \frac{\partial}{\partial x} \\
L_{v^{\prime \prime}}^{v} \equiv\left[\operatorname{Re} U \frac{\partial}{\partial x}-2\left(\frac{\partial^{2}}{\partial x^{2}}+\frac{\partial^{2}}{\partial y^{2}}\right)\right], \\
L_{w}^{v} \equiv \operatorname{Re}\left[-2 \frac{\partial U}{\partial z} \frac{\partial^{2}}{\partial x \partial y}-2 \frac{\partial^{2} U}{\partial y \partial z} \frac{\partial}{\partial x}\right],
\end{gathered}
$$




$$
\begin{gathered}
L_{v}^{\eta} \equiv \operatorname{Re}\left[\frac{\partial^{2} U}{\partial y \partial z}-\frac{\partial U}{\partial y} \frac{\partial}{\partial y}\right], \\
L_{v^{\prime}}^{\eta} \equiv \operatorname{Re} \frac{\partial U}{\partial y}, \\
L_{\eta}^{\eta} \equiv \operatorname{Re} U \frac{\partial}{\partial x}-\left(\frac{\partial^{2}}{\partial x^{2}}+\frac{\partial^{2}}{\partial y^{2}}\right), \\
L_{w}^{\eta} \equiv \operatorname{Re} \frac{\partial^{2} U}{\partial z^{2}} .
\end{gathered}
$$

Here, we note that both $\mathcal{M}$ and $\mathcal{L}$ do not contain any $\partial / \partial z$ for $\psi$, consistent with the derivation of the criterion of absolute instability in section 2.1.

\section{Appendix B. Operators for sensitivity analysis to spanwise wavy base-flow modification}

In this section, we derive the operators used in the sensitivity analysis of complex absolute frequency $\omega_{0}$ to spanwise wavey base-flow modification (section 4.3). We first write the base flow $(2.13)$ as $U(y, z)=U_{0}(y)+A \Delta U(y, z)$. Then, the linearized NavierStokes equation (2.12) is rewritten by splitting it into its counter part with the twodimensional base flow $U_{0}$ and rest of the term corresponding to modification of the linearized operator by $A \Delta U(y, z)$ :

$$
\mathcal{H} \frac{\partial \mathbf{u}}{\partial t}=\mathcal{L}_{2 D} \mathbf{u}+A \mathcal{L}_{3 D} \mathbf{u}
$$

where

$$
\begin{gathered}
\mathcal{H} \equiv\left(\begin{array}{cccc}
1 & 0 & 0 & 0 \\
0 & 1 & 0 & 0 \\
0 & 0 & 1 & 0 \\
0 & 0 & 0 & 0
\end{array}\right), \\
\mathcal{L}_{2 D}\left(-i \frac{\partial}{\partial x},-i \frac{\partial}{\partial z} ; U_{0}\right) \equiv \\
\left(\begin{array}{cccc}
-U_{0} \frac{\partial}{\partial x}+\frac{1}{R e} \nabla^{2} & -\frac{\partial U_{0}}{\partial y} & 0 & -\frac{\partial}{\partial x} \\
0 & -U_{0} \frac{\partial}{\partial x}+\frac{1}{R e} \nabla^{2} & 0 & -\frac{\partial}{\partial y} \\
0 & -U_{0} \frac{\partial}{\partial x}+\frac{1}{R e} \nabla^{2} & -\frac{\partial}{\partial z} \\
\frac{\partial}{\partial y} & \frac{\partial}{\partial z} & 0
\end{array}\right), \\
\mathcal{L}_{3 D}\left(-i \frac{\partial}{\partial x} ; \Delta U\right) \equiv \\
\left.\begin{array}{cccc}
-\Delta U \frac{\partial}{\partial x} & -\frac{\partial \Delta U}{\partial y} & -\frac{\partial \Delta U}{\partial z} & 0 \\
0 & -\Delta U \frac{\partial}{\partial x} & 0 & 0 \\
0 & 0 & -\Delta U \frac{\partial}{\partial x} & -0 \\
0 & 0 & 0 & 0
\end{array}\right)
\end{gathered}
$$

Here, $\mathbf{u}=\left[\begin{array}{lll}u & v & w\end{array}\right]^{T}$ and $\nabla^{2}=\partial^{2} / \partial x^{2}+\partial^{2} / \partial y^{2}+\partial^{2} / \partial z^{2}$. We now consider the normalmode solution of (B 1): $\mathbf{u}(x, y, z)=\hat{\mathbf{q}}(y, z) e^{i(\alpha x+\beta z-\omega t)}$. It is then straightforward to obtain (4.3) in which the operators $\mathcal{L}_{2 D}$ and $\mathcal{L}_{3 D}$ are obtained by substituting $-i \frac{\partial}{\partial x}$ and $-i \frac{\partial}{\partial z}$ in (B 1) respectively with $\alpha$ and $-i \frac{\partial}{\partial z}+\beta$ :

$$
\mathcal{L}_{2 D}\left(-i \frac{\partial}{\partial x},-i \frac{\partial}{\partial z} ; U_{0}\right) \rightarrow \mathcal{L}_{2 D}\left(\alpha,-i \frac{\partial}{\partial z}+\beta ; U_{0}\right)
$$




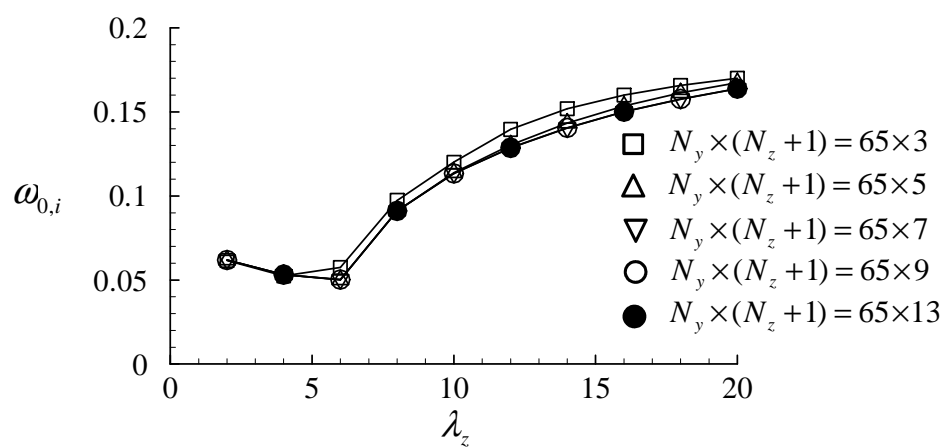

FiguRE 18. Effect of the spanwise grid points $N_{z}$ on the absolute growth rate for varicose base-flow modification $(R e=50$ and $a=1.5)$.

$$
\mathcal{L}_{3 D}\left(-i \frac{\partial}{\partial x} ; \Delta U\right) \rightarrow \mathcal{L}_{3 D}(\alpha ; \Delta U)
$$

We note that when $\Delta U=U_{1}(y) \cos \left(2 \pi z / \lambda_{z}\right)$ as in $(2.13), \mathcal{L}_{3 D}$ does not contain any spanwise unform component.

\section{Appendix C. Convergence with the number of the spanwise grid points}

The number of the spanwise grid points $N_{z}$ is tested to save the computational cost. Figure 18 shows the effect of the spanwise grid points $N_{z}$ for the largest base-flow modification amplitude $A=0.4$ considered. If the number of the spanwise grid points is not enough, the absolute growth rate relatively largely deviates from the converged one for large $\lambda_{z}$. However, the absolute growth rate tends to very rapidly converge with the increase of $N_{z}$, and the converged results are obtained only with $N_{z}=6$. We note that this behavior is probably because the base flow contains only single Fourier mode: for example, even if we consider only a single Fourier mode in Eq. (2.16) for the calculation (i.e. $N_{y} \times\left(N_{z}+1\right)=65 \times 3$ ), the results are not very different from the converged ones. We finally note that, in the case of the sinuous base-modification, the convergence is obtained only with $N_{z}=2$.

\section{REFERENCES}

Del Álamo, J. C. \& JimÉnez, J. 2006 Linear energy amplification in turbulent channels. J. Fluid Mech. 559, 205-213.

Antsaklis, P. J. \& Michel, A. N. 1997 Linear systems. Singapore: McGraw-Hill.

BeARman, P. W. 1965 Investigation of the flow behind a two-dimensional model with a blunt trailing edge and fitted with splitter plates. J. Fluid Mech. 21, 241-255.

Bearman, P. W. 1967 The effect of base bleed on the flow behind a two-dimensional model with a bluent trailing edge. Aero. Q. 18, 207-224.

Bearman, P. W. \& Owen, J. C. 1998 Reduction of bluff-body drag and suppression of vortex shedding by the introduction of wavy separation lines. J. Fluids and Structures 12, 123-130.

Bers, A. 1983 Space-time evolution of plasma instabilities - absolute and convective. In Handbook of Plasma Physics (ed. M. N. Rosenbluth \& R. Z. Sagdeev), , vol. 1, pp. 451-517. North-Holland.

Bertolotti, F. P., Hein, S., Stolte, A. \& Koch, W. 2004 Absolute/convective instability investigation of primary and secondary crossflow vortices. Notes on numerical fluid mechanics and multidisciplinary design 86, 51-62. 
Brancher, P. \& Chomaz, J. M. 1997 Absolute and convective secondary instabilities in spatially periodic shear flows. Phys. Rev. Lett. 78, 658-661.

Brandt, L., Cossu, C., Chomaz, J.-M., Huerre, P. \& Henningson, D. S. 2003 On the convectively unstable nature of optimal streaks in boundary layers. J. Fluid Mech. 485, 221-242.

Brevdo, L. \& Bridges, T. 1996 Absolute and convective instability of spatially periodic flows. Phil. Trans. R. Soc. London A 354, 1027-1064.

Briggs, R. J. 1964 Electron-Stream Interaction with Plasmas. Cambridge, Mass.: MIT Press.

Butler, K. M. \& FARrell, B. F. 1992 Three-dimensional optimal perturbations in viscous shear flow. Phys. Fluids A 4, 1637-1650.

Butler, K. M. \& FArRell, B. F. 1993 Optimal perturbations and streak spacing in wallbounded turbulent shear flow. Phys. Fluids 5, 774-777.

Canuto, C., Hussaini, Y.M., Quarteroni, A. \& Zang, T.A. 1988 Spectral Methods in Fluid Dynamics. New York: Springer.

Carriere, P. \& Monkewitz, P. A. 1999 Convective versus absolute instability in mixed rayleigh-benard-poiseuille convection. J. Fluid Mech. 384, 243 - 262.

Choi, H., Jeon, W.-P. \& Kim, J. 2008 Control of flow over a bluff body. Annu. Rev. Fluid Mech. 40, 113-139.

Chomaz, J. M. 2005 Global instabilities in spatially developing flows: Nonnormality and nonlinearity. Annu. Rev. Fluid Mech. 37, 357-392.

Chomaz, J. M., Huerre, P. \& Redekopp, L. G. 1988 Bifurcations to local and global modes in spatially developing flows. Phys. Rev. Lett. 60, 25-28.

DAREkAR, R. M. \& ShERwin, S. J. 2001 Flow past a square-section cylinder with a wavy stagnation face. J. Fluid Mech. 426, 263-295.

Ellingsen, T. \& Palm, E. 1975 Stability of linear flow. Phys. Fluids 18, 487.

Giannetti, F. \& Luchini, F. 2007 Structural sensitivity of the first instability of the cylinder wake. J. Fluid Mech. 581, 167-197.

Hammond, D. A. \& Redekopp, L. G. 1997 Global dynamics of symmetric and asymmetric wakes. J. Fluid Mech. 331, 231-260.

Herbert, Th. 1988 Secondary instability of boundary-layers. Annu. Rev. Fluid Mech. 20, 487526.

Huerre, P. \& Monkewitz, P. A. 1985 Absolute and convective instabilities in free shear layers. J. Fluid Mech. 159, 151-168.

Huerre, P. \& Monkewitz, P. A. 1990 Local and global instabilities in spatially developing flows. Annu. Rev. Fluid Mech. 22, 473-537.

Huerre, P. \& Rossi, M. 1998 Hydrodynamic instabilities in open flows. In Hydrodynamic and Nonlinear Instabilities (ed. C. Godrèche \& P. Manneville), pp. 81-294. Cambridge (UK): Cambridge University Press.

HwANG, Y. \& CHOI, H. 2006 Control of absolute instability by basic-flow modification in a parallel wake at low reynolds number. J. Fluid Mech. 560, 465-475.

HwANG, Y. \& CHOI, H. 2008 Sensitivity of global instability of spatially developing flow in weakly and fully nonlinear regimes. Phys. Fluid 20, 071703.

Hwang, Y. \& Cossu, C. 2010 a Amplification of coherent streaks in the turbulent Couette flow: an input-output analysis at low Reynolds number. J. Fluid Mech. 643, 333-348.

Hwang, Y. \& Cossu, C. $2010 b$ Linear non-normal energy amplification of harmonic and stochastic forcing in the turbulent channel flow. J. Fluid Mech. 664, 51-73.

JACKSON, C. P. 1987 A finite-element study of the onset of vortex shedding in flow past variously shaped bodies. J. Fluid Mech. 182, 23-45.

KIM, J. 2005 Distributed forcing of flow over a circular cylinder. PhD thesis, School of Mechanical and Aerospace Engineering, Seoul National University, Seoul, Korea.

Kim, J. \& Chor, H. 2005 Distributed forcing of flow over a circular cylinder. Phys. Fluids 17, 033133.

Kim, J., Hahn, S., Kim, J., Lee, D., Choi, J., Jeon, W.-P. \& Choi, H. 2004 Active control of turbulent flow over a model vehicle for drag reduction. J. Turbulence $\mathbf{5}, 019$.

Kwon, K. \& CHOI, H. 1996 Control of laminar vortex shedding behind a circular cylinder using splitter plates. Phys. Fluids 8, 478-496. 
LANDAhL, M. T. 1980 A note on an algebraic instability of inviscid parallel shear flows. J. Fluid Mech. 98, 243-251.

LEU, T.-S. \& Ho, C.-M. 2000 Control of global instability in a non-parallel near wake,. Astron. Astrophys. 404, 345-378.

Marquet, O., Sipp, D. \& Jacquin, L. 2008 Sensitivity analysis and passive control of cylinder flow. J. Fluid Mech. 615, 221-252.

Mathis, C., Provansal, M. \& Boyer, L. 1984 The Bénard-von Kármán instability: an experimental study near the threshold. J. Phys. Paris Lett. 45, 483-491.

Min, C. \& Choi, H. 1999 Suboptimal feedback control of vortex shedding at low reynolds numbers. J. Fluid Mech. 401, 123-156.

Monkewitz, P.A. 1988 The absolute and convective nature of instability in two-dimensional wakes at low Reynolds numbers. Phys. Fluids 31, 999-1006.

Monkewitz, P.A., Huerre, P. \& Chomaz, J.M. 1993 Global linear stability analysis of weakly non-parallel shear flows. J. Fluid Mech. 251, 1-20.

Nishioka, M. \& Sato, H. 1974 Measurements of velocity distributions in the wake of a circular cylinder at low reynolds numbers. J. Fluid Mech. 765, 97-112.

Owen, J. C., Szewczyk, A. A. \& Bearman, P. W. 2000 Suppression of kármán vortex shedding. Phys. Fluids 12, S9.

PARK, H., Jeon, W.-P., H.Choi \& Yoo, J. Y. 2006 Drag reduction in flow over a twodimensional bluff body with a blunt trailing edge using a new passive device. J. Fluid Mech. 563, 389-414.

Park, J., Hwang, Y. \& Cossu, C. 2011 On the stability of large-scale streaks in the turbulent Couette and Poiseulle flows. C. R. Mècanique 339 (1), 105103.

PIER, B 2002 On the frequency selection of finite-amplitude vortex shedding in the cylinder wake. J. Fluid Mech. 458, 407-417.

PIER, B 2007 Primary crossflow vortices, secondary absolute instabilities and their control in the rotating-disk boundary layer. J. Eng. Math. 57, 237-251.

Pralits, J. O., Brandt, L. \& Giannetti, F. 2010 Instability and sensitivity of the flow around a rotating circular cylinder. J. Fluid Mech. 650, 513-536.

Provansal, M., Mathis, C. \& Boyer, L. 1987 Bénard-von Kármán instability: Transient and forced regimes. J. Fluid Mech. 182, 1-22.

Pujals, G., García-Villalba, M., Cossu, C. \& Depardon, S. 2009 A note on optimal transient growth in turbulent channel flows. Phys. Fluids 21, 015109.

Reddy, S. C. \& Henningson, D. S. 1993 Energy growth in viscous channel flows. J. Fluid Mech. 252, 209-238.

Reddy, S. C., Schmid, P. J., Baggett, J. S. \& Henningson, D. S. 1998 On the stability of streamwise streaks and transition thresholds in plane channel flows. J. Fluid Mech. 365, 269-303.

Rodriguez, O. 1991 Base drag reduction by the control of three-dimensional unsteady vortical structures. Exp. Fluids 11, 218-22.

Roshko, A. 1955 On the wake and drag of bluff bodies. J. Aeronaut. Sci. 22, 12.

Schmid, P. J. \& Henningson, D. S. 2001 Stability and Transition in Shear Flows. New York: Springer.

Schoppa, W. \& Hussain, F. 2002 Coherent structure generation in near-wall turbulence. J. Fluid Mech. 453, 57-108.

Schumm, M., Berger, E. \& Monkewitz, P. A. 1994 Self-excited oscillations in the wake of two-dimensional bluff bodies and their control. J. Fluid Mech. 271, 17-53.

Son, D., JEON, S. \& CHOI, H. 2011 A proportional-integral-differential control of flow over a circular cylinder. Phil. Trans. R. Soc. A 369 (1940), 1540-1555.

Stansby, P. K. 1974 The effects of end plates on the base pressure coefficient of a circular cylinder. Aerosp. J. 78, 36-37.

Strykowski, P. J. \& Sreenivasan, K. R. 1990 On the formation and suppression of vortex 'shedding' at low Reynolds number. J. Fluid Mech. 218, 71-107.

TAnner, M. 1972 A method of reducing the base drag of wings with blunt trailing edges. Aero. Q. 23, 15-23.

Tombazis, N. \& Bearman, P. W. 1997 A study of three dimensional aspects of vortex shedding from a bluff body with a mild geometric disturbance. J. Fluid Mech. 330, 85-112. 
Waleffe, F. 1995 Hydrodynamic stability and turbulence: Beyond transients to a selfsustaining process. Stud. Appl. Math. 95, 319-343.

Williamson, C. H. K. 1996 Vortex dynamics in the cylinder wake. Annu. Rev. Fluid Mech. 28, 477-539.

Wood, C. J. 1967 The effect of base bleed on a periodic wake. J. Aeronaut. Soc. 68, 477-482. ZeBIB, A. 1987 Stability of viscous flow past a circular cylinder. J. Eng. Math. 21, 155-165. 